TRANSACTIONS OF THE

AMERICAN MATHEMATICAL SOCIETY

Volume 357, Number 5, Pages 1801-1828

S 0002-9947(04)03811-5

Article electronically published on December 29, 2004

\title{
EXISTENCE AND ASYMPTOTIC BEHAVIOR FOR A SINGULAR PARABOLIC EQUATION
}

\author{
JUAN DÁVILA AND MARCELO MONTENEGRO
}

\begin{abstract}
We prove global existence of nonnegative solutions to the singular parabolic equation $u_{t}-\Delta u+\chi_{\{u>0\}}\left(-u^{-\beta}+\lambda f(u)\right)=0$ in a smooth bounded domain $\Omega \subset \mathbb{R}^{N}$ with zero Dirichlet boundary condition and initial condition $u_{0} \in C(\Omega), u_{0} \geq 0$. In some cases we are also able to treat $u_{0} \in L^{\infty}(\Omega)$. Then we show that if the stationary problem admits no solution which is positive a.e., then the solutions of the parabolic problem must vanish in finite time, a phenomenon called "quenching". We also establish a converse of this fact and study the solutions with a positive initial condition that leads to uniqueness on an appropriate class of functions.
\end{abstract}

\section{INTRODUCTION}

In this paper we are concerned with nonnegative solutions to the singular parabolic problem

$$
\left\{\begin{aligned}
u_{t}-\Delta u+\chi_{\{u>0\}} g(u) & =0 & & \text { in } \Omega \times(0, T), \\
u(x, t) & =0 & & \text { on } \partial \Omega \times(0, T), \\
u(x, 0) & =u_{0}(x) & & \text { in } \Omega,
\end{aligned}\right.
$$

where $0<T<\infty$ or $T=\infty$ and $\Omega \subset \mathbb{R}^{N}$ is a bounded smooth domain. The nonlinearity $g(u)$ is singular at $u=0$ and is defined by

$$
g(u)=u^{-\beta}-f(u)
$$

where $0<\beta<1$ and $f:[0, \infty) \rightarrow[0, \infty)$ satisfies

$$
f \geq 0 \text { is } C^{2} \text { in }[0, \infty)
$$

and

$$
f(u) \leq C(1+u) \quad \forall u \geq 0,
$$

where $C \geq 0$ is a constant. We denote the characteristic function of the set $\{x \in$ $\Omega \mid u(x)>0\}$ by $\chi_{\{u>0\}}$ and we tacitly assume $\chi_{\{u>0\}} g(u)=0$ whenever $u=0$.

Equation $(P)$ is a limiting situation of models describing enzymatic kinetics [B] and in the Langmuir-Hinshelwood model of heterogeneous chemical catalyst kinetics [A]; see also [D]. It has been studied already in DL, FLV, FH, FK, L, $[\mathrm{P}]$. These authors have addressed questions about existence and the qualitative behavior of these solutions. The most striking phenomenon that can occur under some circumstances is that, even starting with a positive initial condition, a solution

Received by the editors July 18, 2003.

2000 Mathematics Subject Classification. Primary 35B40, 35K55.

Key words and phrases. Singular parabolic equation, quenching problem. 
may vanish in finite time. This behavior is called "quenching" and was first observed in the pioneering paper $[\mathrm{K}$.

The main novelty is that our problem has zero Dirichlet boundary condition which introduces new difficulties. We are concerned with the existence of a solution and we study its regularity and asymptotics as $t \rightarrow \infty$. Another difference with the previous works is the nonlinearity $f(u)$. In general the uniqueness of solutions still remains open. However, we are able to obtain uniqueness in a particular class of positive solutions.

We adopt the following definition of a solution to $(P)$. Let $T>0$ and $u_{0} \in$ $L^{\infty}(\Omega), u_{0} \geq 0$. By a solution of $P$ we mean a function $u \in L^{\infty}(\Omega \times(0, T))$, $u \geq 0$ such that

$$
\chi_{\{u>0\}} g(u) \in L^{1}(\Omega \times(0, T))
$$

and

$$
\int_{\Omega} u_{0} \varphi(0) d x+\int_{0}^{T} \int_{\Omega}\left(\varphi_{t}+\Delta \varphi\right) u-\chi_{\{u>0\}} g(u) \varphi d x d t=0
$$

for every $\varphi \in C^{2}(\bar{\Omega} \times[0, T])$ with $\varphi=0$ on $\partial \Omega \times(0, T)$ and $\varphi(T)=0$ in $\Omega$.

A global solution in $(0, \infty)$ is a function $u \geq 0$ such that $u \in L^{\infty}(\Omega \times(0, T))$ for all $T>0$ and satisfies (3) and (4) for every $T>0$.

We show that a solution to $(P)$ exists by considering the perturbed parabolic problem $\left(P_{\varepsilon}\right)$ :

$$
\left\{\begin{aligned}
u_{t}^{\varepsilon}-\Delta u^{\varepsilon}+g_{\varepsilon}\left(u^{\varepsilon}\right) & =0 & & \text { in } \Omega \times(0, T), \\
u^{\varepsilon}(x, t) & =0 & & \text { on } \partial \Omega \times(0, T), \\
u^{\varepsilon}(x, 0) & =u_{0}(x) & & \text { in } \Omega,
\end{aligned}\right.
$$

where, for $\varepsilon>0$,

$$
g_{\varepsilon}(u)=\frac{u}{(u+\varepsilon)^{1+\beta}}-f(u) .
$$

Standard results for semilinear parabolic equations guarantee that for any initial condition $u_{0} \in L^{\infty}(\Omega), u_{0} \geq 0$ there is a unique solution $u^{\varepsilon} \in L^{\infty}(\Omega \times(0, T))$ of $\left(P_{\varepsilon}\right)$; see [LSU]. Moreover, $u^{\varepsilon}$ is $C^{3}$ by [LSU] and positive in $\Omega \times(0, T)$. We manage to pass the limit $u^{\varepsilon} \rightarrow u$ as $\varepsilon \rightarrow 0$ and show that $u$ is a genuine solution of $(P)$; this is the content of our main result.

Theorem 1.1. Let $u_{0} \in L^{\infty}(\Omega), u_{0} \geq 0$ and assume (11) and (2). Then the solution $u^{\varepsilon}$ of $\left[P_{\varepsilon}\right)$ converges uniformly on compact subsets of $\Omega \times(0, T]$ as $\varepsilon \rightarrow 0$ to a function

$$
u=\lim _{\varepsilon \rightarrow 0} u^{\varepsilon} .
$$

If, moreover, $u_{0} \in C(\Omega)$, then $u \in C(\Omega \times[0, T])$ and $u$ is a solution of the problem $(\mathbb{P})$ in the sense of (3) - (4).

Remark 1.2. In Theorem 1.1 one can replace the assumption $u_{0} \in C(\Omega)$ by weaker ones and still deduce that $u=\lim _{\varepsilon \rightarrow 0} u^{\varepsilon}$ is a solution to (P) in the sense of (3i)-(4). Here are two such conditions:

i) $u_{0}$ satisfies $u_{0} \geq c \delta^{\nu}$ for some $c>0$ and $1<\nu<\frac{2}{1+\beta}$, where $\delta$ stands for the distance function to the boundary

$$
\delta(x)=\operatorname{dist}(x, \partial \Omega),
$$


or

ii) $u_{0}$ can be approximated from below by nonnegative continuous functions, that is, there exists a sequence $\left(u_{0}^{j}\right)$ in $C(\Omega)$ such that $0 \leq u_{0}^{j} \leq u_{0}$ and $u_{0}^{j} \rightarrow u_{0}$ a.e.

If, merely, $u_{0} \in L^{\infty}(\Omega), u_{0} \geq 0$, we deduce that (3) still holds and that

$$
\int_{\Omega} u(\tau) \varphi(\tau) d x+\int_{\tau}^{T} \int_{\Omega}\left(\varphi_{t}+\Delta \varphi\right) u-\chi_{\{u>0\}} g(u) \varphi d x d t=0
$$

for every $\tau>0$ and all $\varphi \in C^{2}(\bar{\Omega} \times[0, T])$ with $\varphi=0$ on $\partial \Omega \times(0, T)$ and $\varphi(T)=$ 0 in $\Omega$. These statements are proved in Section 4. This means that $u$ satisfies $u_{t}-\Delta u+\chi_{\{u>0\}} g(u)=0$ in $\Omega \times(0, T)$, and $u=0$ in $\partial \Omega \times(0, T)$, but it remains an open question whether it satisfies the initial condition.

The core idea to prove Theorem 1.1 is to obtain uniform estimates for $u^{\varepsilon}$ which are independent of $\varepsilon$. This approach was employed by Phillips $[\mathrm{P}$ for a similar parabolic problem posed in $\mathbb{R}^{N}$ with a compactly supported initial data or on a bounded domain with positive boundary data. Our estimate in space is inspired by the one in $\mathrm{P}$ but it is more involved because of the zero Dirichlet boundary condition; see Section 2. The estimate in time is obtained using a scaling argument, used in DM1 also, which allows us to obtain optimal Hölder regularity in time. This can be seen clearly in [FK2] where they treat a problem related to ours. As a byproduct of these estimates we obtain

Theorem 1.3. Let $u_{0} \in L^{\infty}(\Omega), u_{0} \geq 0$ and assume (1) and (2). Then $u^{\varepsilon}$ satisfies: for any $0<\tau<T$ and $\Omega^{\prime} \subset \subset \Omega$ there exists $C$ such that

$$
\left|u^{\varepsilon}(x, t)-u^{\varepsilon}(x, s)\right| \leq C|t-s|^{1 /(1+\beta)} \quad \forall x \in \Omega^{\prime}, \forall t, s \in(\tau, T)
$$

and

$$
\left|\nabla u^{\varepsilon}(x, t)-\nabla u^{\varepsilon}(y, t)\right| \leq C|x-y|^{\frac{1-\beta}{1+\beta}} \quad \forall x, y \in \Omega^{\prime}, \forall t \in(\tau, T),
$$

where $C$ is independent of $\varepsilon$. If, moreover, $u_{0} \in C^{2}(\Omega)$, then one can replace $(\tau, T)$ with $[0, T)$ in the above estimates. The limit $u=\lim _{\varepsilon \rightarrow 0} u^{\varepsilon}$ also satisfies estimates (7) and (8).

Next we point out some connections of problem $P$ with its stationary version. In dealing with the elliptic problem it is convenient to replace the nonlinearity $f(u)$ by $\lambda f(u)$ where $\lambda>0$ is a parameter. Thus we consider

$$
\left\{\begin{aligned}
-\Delta u & =\chi_{\{u>0\}}\left(-u^{-\beta}+\lambda f(u)\right) & & \text { in } \Omega, \\
u & \geq 0 & & \text { in } \Omega, \\
u & =0 & & \text { on } \partial \Omega .
\end{aligned}\right.
$$

From now on we assume that $f$ satisfies (11) and is

$$
\text { concave, increasing and } \lim _{u \rightarrow \infty} \frac{f(u)}{u}=0 \text {. }
$$

We say that a function $u \in L^{\infty}(\Omega), u \geq 0$ is a solution of $(E)$ if $\chi_{\{u>0\}} g(u) \in L^{1}(\Omega)$ and

$$
\int_{\Omega} u \Delta \varphi-\chi_{\{u>0\}} g(u) \varphi=0
$$

for all $\varphi \in C^{2}(\Omega)$ with $\varphi=0$ on $\partial \Omega$. 
Under these hypotheses in [DM1] we proved the following result.

Theorem 1.4. Assume (1) and (9). Then for any $\lambda>0$, problem (E) possesses a unique maximal solution $u_{\lambda}$. Moreover, there exists a dividing value $\lambda^{*} \in(0, \infty)$ such that for $\lambda>\lambda^{*}$ the maximal solution $u_{\lambda}$ is positive in $\Omega$, while for $0<\lambda<\lambda^{*}$ the set $\left\{u_{\lambda}=0\right\}$ has positive measure and thus $u_{\lambda}$ possesses a free boundary.

If $\lambda>\lambda^{*}$ one has in fact $a \delta \leq u_{\lambda} \leq b \delta$ in $\Omega$ where $a, b$ are positive constants. By a result in [GL we conclude that $u_{\lambda} \in C^{1,1-\beta}(\bar{\Omega})$ for $\lambda>\lambda^{*}$. For $0<\lambda \leq \lambda^{*}$ the maximal solution $u_{\lambda}$ has optimal regularity $C^{1, \frac{1-\beta}{1+\beta}}(\bar{\Omega})$ (see DM1, Da]). We also prove in [DM1] that for $\lambda=\lambda^{*}$ the maximal solution is positive a.e. and there are examples in DM2] showing that it can vanish at some points inside $\Omega$. But for $0 \leq \lambda<\lambda^{*}$ all solutions of $[E]$ must vanish on a set of positive measure.

Regarding the asymptotic behavior of solutions to $P$ we are motivated by a result in [FLV] that says that every accumulation point of the orbit $u(t)$ of a solution to $(P]$ is a solution of the stationary problem. Although the result in [FLV] was established for a problem with a positive boundary condition it still holds in our context.

Proposition 1.5. Assume that $f$ satisfies (1) and (9). Let $u_{0} \in L^{\infty}(\Omega), u_{0} \geq 0$, and let $u$ denote the solution of $(P)$ of Theorem [1.1. Then the orbits of u are compact in $C(\Omega)$ (with respect to uniform convergence on compact sets) and any limit point is a solution of the elliptic equation (E).

In view of Theorem 1.4 we reach the following conclusion: if $\lambda<\lambda^{*}$ and if $u\left(t_{k}\right)$ converges for a sequence $t_{k} \rightarrow \infty$, then the limit $u=\lim _{t_{k} \rightarrow \infty} u\left(t_{k}\right)$ has to vanish somewhere in $\Omega$. Thus there exists a point $x_{0} \in \Omega$ such that $u\left(t_{k}, x_{0}\right) \rightarrow 0$ as $t_{k} \rightarrow \infty$. In other words, this means that "quenching" occurs at least in infinite time. We strengthen this assertion, by proving that in fact "quenching" must happen in finite time.

Theorem 1.6. Assume (19), (9) and that $0 \leq \lambda<\lambda^{*}$. Then every solution of $(\underline{P})$ vanishes in finite time, in the sense that the measure of the vanishing set is $|\{(x, t) \in \Omega \times(0, \infty) \mid u(x, t)=0\}|>0$.

Actually, what we will prove is that if the parabolic problem $(\mathbb{P})$ has a solution that is positive a.e., then the elliptic problem (ED) has a solution which is positive a.e. There is a sort of converse of this result.

Theorem 1.7. Assume (19), (9) and suppose that the elliptic problem (E) has a solution $w$ which is positive a.e. Assume that the initial data $u_{0} \in L^{\infty}(\Omega)$ satisfies $u_{0} \geq w, u_{0} \not \equiv w$. Then the solution $u$ of Theorem 1.1 satisfies $(P)$ in the sense of (3) -(4) and there exists a continuous function $c:(0, \infty) \rightarrow(0, \infty)$ such that

$$
u(t) \geq c(t) \delta \quad \forall t \in(0, \infty) .
$$

Note that we assume only that the initial condition $u_{0} \geq w$ and $u_{0} \not \equiv w$, and the theorem allows us to conclude that $u=\lim _{\varepsilon \rightarrow \infty} u^{\varepsilon}$ satisfies $(\underline{P}$. This complements the statement of Remark 1.2 and is especially interesting in the case when the solution $w$ of $(E)$ vanishes at some points but is positive a.e. We already mentioned that examples of this situation exist (see [DM2]). By comparison $u(t) \geq w$, but this inequality for itself does not prevent $u(t)$ from "quenching" in finite time. The 
content of the previous theorem is precisely that $u(t)$ does not vanish in finite time (although it can "quench" in infinite time).

There is some parallel between Theorems[1.6] and 1.7] and the results of [BCMR], in spite of the completely different kind of nonlinearities treated there. They consider a semilinear parabolic equation with a smooth superlinear nonlinearity, and study the relation between the existence of global solutions of the parabolic equation and existence of "weak" solutions for the stationary problem.

So far we have always considered $0<\beta<1$ but the techniques applied before permit us to claim that Theorem 1.7 is sharp with respect to $\beta$.

Corollary 1.8. Assume (11) and (9). If $\beta \geq 1$, there is no positive global classical solution of $(\underline{P})$.

Finally, we mention some results concerning the case of an initial data $u_{0}$ which is positive in $\Omega$, and in fact we shall assume a stronger condition, namely

$$
u_{0} \geq c \delta^{\nu}
$$

where $c>0,1<\nu<\frac{2}{1+\beta}$; see Remark [1.2. Our interest in these initial conditions is twofold. On one hand, we need to consider such $u_{0}$ when studying the asymptotic behavior of solutions of $(P)$. On the other hand, we do not know in general whether the solution to $(P$ is unique. However, uniqueness holds in a suitable class of solutions which is related to condition (10).

We begin by observing that if $u_{0}$ satisfies (10), then the solution $u$ of Theorem 1.1 stays positive for some time.

Lemma 1.9. Assume (1) and (2). Let $u_{0} \in L^{\infty}(\Omega)$ be such that $u_{0} \geq c \delta^{\nu}$ for some $c>0$ and $1<\nu<\frac{2}{1+\beta}$. Then the solution $u$ of Theorem 1.1 of $(P$ satisfies $u(t) \geq c^{\prime} \delta^{\nu}$ in $(0, T)$ for some $c^{\prime}>0$ and $T$, both of which depend on $c$ and $\nu$.

Solutions in an adequate class are unique.

Theorem 1.10. Assume (11), (2) and $u_{0} \in L^{\infty}(\Omega)$, and let $u_{0} \geq c \delta^{\nu}$ for some $c>0$ and $1<\nu<\frac{2}{1+\beta}$. Then $(\mathbb{P})$ has at most one solution in the set

$$
\begin{array}{r}
\mathcal{M}=\left\{u \in L^{\infty}(\Omega \times(0, T)): \forall T^{\prime} \in(0, T) \text { there exists } c>0\right. \\
\text { such that } \left.u(t) \geq c \delta^{\nu} \text { for all } t \in\left(0, T^{\prime}\right)\right\} .
\end{array}
$$

We obtain the above result as an immediate consequence of the following comparison principle stated in terms of the heat semigroup $S(t)$ with zero Dirichlet boundary condition.

Lemma 1.11. Under assumptions (1) and (2), let $u, v \in L^{\infty}(\Omega \times(0, T))$ be a subsolution and a supersolution of $(P)$ respectively, in the sense that they satisfy $\chi_{\{u>0\}} g(u), \chi_{\{v>0\}} g(v) \in L^{1}(\Omega \times(0, T))$ and

$$
\begin{array}{ll}
u(t) \leq S(t) u_{0}-\int_{0}^{t} S(t-s)\left(\chi_{\{u(s)>0\}} g(u(s))\right) d s & t \in[0, T], \\
v(t) \geq S(t) v_{0}-\int_{0}^{t} S(t-s)\left(\chi_{\{v(s)>0\}} g(v(s))\right) d s & t \in[0, T] .
\end{array}
$$

Furthermore, assume that there exists $c>0$ and $1<\nu<\frac{2}{1+\beta}$ such that the supersolution $v$ satisfies

$$
v(t) \geq c \delta^{\nu} \quad \text { for } t \in(0, T)
$$


Then, if $u(0) \leq v(0)$, we have

$$
u(t) \leq v(t) \quad \text { for } t \in(0, T) .
$$

The main ingredient in the proof of Lemma 1.11 is a version of the smoothing effect for the heat semigroup $S(t)$ with zero Dirichlet boundary condition, which involves weights of the form $\delta^{m}$.

Proposition 1.12. For any $m \in[0,1]$ and $t>0$ the mapping from $L^{2}(\Omega) \rightarrow L^{2}(\Omega)$ given by $\varphi \mapsto S(t)\left(\delta^{-m} \varphi\right)$ is continuous and, moreover,

$$
\left\|S(t)\left(\delta^{-m} \varphi\right)\right\|_{L^{2}(\Omega)} \leq C t^{-m / 2}\|\varphi\|_{L^{2}(\Omega)}
$$

for every $\varphi \in L^{2}(\Omega)$, where $C$ depends only on $\Omega$ and $m$.

Finally, we mention works dealing with other questions in similar problems. For example the blow up of $u_{t}$ at the time of quenching has been considered in various situations; see $\mathrm{AK}, \mathrm{DL}$ and $\mathrm{CK}$ when $\beta \geq 1$. There are results on the blow up of $\Delta u$ in one dimension in [FKL] and in higher dimensions for radial solutions FK2]. There are also some works in which the profile of the vanishing solution is studied in the case of dimension 1 and for radial solutions in higher dimensions; see Gu, FH, FHQ, FK2, FG].

The paper is organized as follows. In Sections 2 and 3 we obtain the necessary estimates for the solution $u^{\varepsilon}$ of $\left[P_{\varepsilon}\right.$, which we apply in Section 4 in the proof of Theorem 1.1. That section contains also proofs for the statements of Remark 1.2 regarding a weakening of the hypothesis $u_{0} \in C(\Omega)$. Then, before going into the proof of Theorems [1.6] and [1.7] in Section [5 we present proofs for the results concerning the case of an initial condition $u_{0}$ satisfying (10): Lemmas 1.9 1.11 and Proposition 1.12, In Section 6 we prove Theorems 1.6, [1.7] and Corollary [1.8.

\section{Estimates IN SPACE FOR THE APPROXIMATE SOLUTION}

Our first goal is to obtain a local estimate for our approximate solution $u^{\varepsilon}$. The result below is a weighted estimate of the gradient of $u^{\varepsilon}$ in $\Omega$ where the weight $\psi$ has the following properties:

$$
\psi \in C^{2}(\bar{\Omega}), \psi>0 \text { in } \Omega, \psi=0 \text { on } \partial \Omega \text { and } \frac{|\nabla \psi|^{2}}{\psi} \text { is bounded in } \Omega .
$$

Lemma 2.1. Let $T>0$ and $u_{0} \in C^{1}(\bar{\Omega})$ be such that $u_{0} \geq 0$. Suppose that $u^{\varepsilon} \in C^{3}(\Omega \times(0, T]) \cap C^{1}(\bar{\Omega} \times[0, T])$ satisfies

$$
\begin{array}{rlrl}
u_{t}^{\varepsilon}-\Delta u^{\varepsilon}+g_{\varepsilon}\left(u^{\varepsilon}\right) & =0 & & \text { in } \Omega \times(0, T), \\
u^{\varepsilon} & \geq 0 & \text { in } \Omega \times(0, T), \\
u^{\varepsilon}(x, 0) & =u_{0}(x) & \text { in } \Omega .
\end{array}
$$

Then there is a constant $C_{1}>0$ independent of $\varepsilon$ such that

$$
\frac{\psi(x)\left|\nabla u^{\varepsilon}(x, t)\right|^{2}}{u^{\varepsilon}(x, t)^{1-\beta}+u^{\varepsilon}(x, t)} \leq \max \left(C_{1}, \sup _{\Omega} \frac{\psi\left|\nabla u_{0}\right|^{2}}{u_{0}^{1-\beta}+u_{0}}\right) \quad \forall x \in \Omega, t \in[0, T] .
$$

$C_{1}$ depends only on $\Omega, N, \beta, \psi,\left\|u^{\varepsilon}\right\|_{L^{\infty}(\Omega \times(0, T))}, f$ and $f^{\prime}$.

Remark 2.2. One can in fact choose $C_{1}$ independently of $\varepsilon$; see Step 1 in Section 4 
Proof. In the course of the proof we denote $u=u^{\varepsilon}$. Let

$$
Z(u)=u^{1-\beta}+u
$$

and consider the functions

$$
w=\frac{|\nabla u|^{2}}{Z(u)}, \quad v=w \psi
$$

We will prove the estimate (16) by contradiction, so we assume that (16) fails, i.e., that

$$
\sup _{\Omega \times(0, T)} v>\max \left(C_{1}, \sup _{\Omega} \frac{\psi\left|\nabla u_{0}\right|^{2}}{u_{0}^{1-\beta}+u_{0}}\right),
$$

where $C_{1}>0$ will be fixed later independently of $\varepsilon$.

Note that $v$ is continuous in $\bar{\Omega} \times[0, T]$, and therefore it attains its maximum at some point $\left(x_{0}, t_{0}\right) \in \bar{\Omega} \times[0, T]$. Thus, by (19)

$$
v\left(x_{0}, t_{0}\right)>\max \left(C_{1}, \sup _{\Omega} \frac{\psi\left|\nabla u_{0}\right|^{2}}{u_{0}^{1-\beta}+u_{0}}\right) .
$$

Then $x_{0} \in \Omega$, because $v=0$ on $\partial \Omega$ and $t_{0}>0$ by (20). Hence

$$
\nabla v\left(x_{0}, t_{0}\right)=0
$$

and

$$
\Delta v\left(x_{0}, t_{0}\right)-v_{t}\left(x_{0}, t_{0}\right) \leq 0 .
$$

Our aim is to compute $\Delta v-v_{t}$ and evaluate at $\left(x_{0}, t_{0}\right)$. As we shall see this leads to the absurd $\Delta v\left(x_{0}, t_{0}\right)-v_{t}\left(x_{0}, t_{0}\right)>0$ if one fixes $C_{1}$ large enough. Let us proceed with the computations:

$$
\begin{aligned}
\Delta v-v_{t} & =\psi \Delta w+2 \nabla w \nabla \psi+w \Delta \psi-\psi w_{t} \\
& =\psi\left(\Delta w-w_{t}\right)+w \Delta \psi+2 \nabla w \nabla \psi .
\end{aligned}
$$

The derivatives of $w$ are (where the convention of summation over repeated indices is adopted)

$$
\begin{aligned}
\partial_{i} w & =\frac{2 \partial_{j} u \partial_{i j} u Z(u)-|\nabla u|^{2} Z^{\prime}(u) \partial_{i} u}{Z(u)^{2}}, \\
w_{t} & =\frac{2 \partial_{j} u \partial_{j}\left(u_{t}\right) Z(u)-|\nabla u|^{2} Z^{\prime}(u) u_{t}}{Z(u)^{2}}
\end{aligned}
$$

and

$$
\begin{aligned}
\Delta w=\partial_{i i} w= & \frac{2\left(\partial_{i j} u\right)^{2} Z(u)+2 \partial_{j} u \partial_{j}(\Delta u) Z(u)-|\nabla u|^{4} Z^{\prime \prime}(u)-|\nabla u|^{2} Z^{\prime}(u) \Delta u}{Z(u)^{2}} \\
& -2 \frac{Z^{\prime}(u)}{Z(u)} \partial_{i} u \partial_{i} w .
\end{aligned}
$$


Therefore,

$$
\begin{aligned}
& \Delta w-w_{t} \\
& =\frac{2\left(\partial_{i j} u\right)^{2} Z(u)+2 \partial_{j} u \partial_{j}\left(\Delta u-u_{t}\right) Z(u)-|\nabla u|^{4} Z^{\prime \prime}(u)-|\nabla u|^{2} Z^{\prime}(u)\left(\Delta u-u_{t}\right)}{Z(u)^{2}} \\
& \quad-2 \frac{Z^{\prime}(u)}{Z(u)} \partial_{i} u \partial_{i} w,
\end{aligned}
$$

and using equation (13) we obtain

$$
\begin{aligned}
\Delta w-w_{t}= & \frac{2\left(\partial_{i j} u\right)^{2} Z(u)+2|\nabla u|^{2} Z(u) g_{\varepsilon}^{\prime}(u)-|\nabla u|^{4} Z^{\prime \prime}(u)-|\nabla u|^{2} Z^{\prime}(u) g_{\varepsilon}(u)}{Z(u)^{2}} \\
& -2 \frac{Z^{\prime}(u)}{Z(u)} \partial_{i} u \partial_{i} w .
\end{aligned}
$$

From now on all functions appearing in the expressions below are evaluated at the point $\left(x_{0}, t_{0}\right)$ or just $x_{0}$ in the case of $\psi$. Relation (21) provides

$$
\psi \nabla w+w \nabla \psi=0
$$

and hence

$$
\nabla w \nabla \psi=-w \frac{|\nabla \psi|^{2}}{\psi}
$$

Substituting in (23),

$$
\Delta v-v_{t}=\psi\left(\Delta w-w_{t}\right)+w\left(\Delta \psi-2 \frac{|\nabla \psi|^{2}}{\psi}\right) .
$$

Inserting (25) in (26),

$$
\begin{aligned}
\Delta v-v_{t}= & \psi\left(\Delta w-w_{t}\right)+w\left(\Delta \psi-2 \frac{|\nabla \psi|^{2}}{\psi}\right) \\
= & \psi\left[\frac{2\left(\partial_{i j} u\right)^{2} Z(u)+2|\nabla u|^{2} g_{\varepsilon}^{\prime}(u) Z(u)-|\nabla u|^{4} Z^{\prime \prime}(u)-|\nabla u|^{2} Z^{\prime}(u) g_{\varepsilon}(u)}{Z(u)^{2}}\right. \\
& \left.-2 \frac{Z^{\prime}(u)}{Z(u)} \partial_{i} u \partial_{i} w\right]+w\left(\Delta \psi-2 \frac{|\nabla \psi|^{2}}{\psi}\right)
\end{aligned}
$$

which is equivalent to

$$
\begin{gathered}
\Delta v-v_{t}=\frac{1}{Z(u)}\left[2 \psi\left(\partial_{i j} u\right)^{2}+2 \psi Z(u) g_{\varepsilon}^{\prime}(u) w-\psi Z(u) Z^{\prime \prime}(u) w^{2}-\psi w g_{\varepsilon}(u) Z^{\prime}(u)\right. \\
\left.-2 \psi Z^{\prime}(u) \partial_{i} u \partial_{i} w\right]+w\left(\Delta \psi-2 \frac{|\nabla \psi|^{2}}{\psi}\right)
\end{gathered}
$$

Without loss of generality, we are going to assume that $\nabla u\left(x_{0}, t_{0}\right)$ is parallel to the first coordinate axis. Then from (21) we have

$$
\partial_{1} v\left(x_{0}, t_{0}\right)=0 .
$$

By virtue of (24) we obtain the expression

$$
\partial_{11} u=\frac{1}{2} w\left(Z^{\prime}(u)-\frac{\partial_{1} \psi}{\psi \partial_{1} u} Z(u)\right)
$$


which, after substitution in (27), yields

$$
\begin{aligned}
& \Delta v-v_{t} \geq \frac{1}{Z(u)}\left[\frac{1}{2} \psi w^{2}(\right.\left.Z^{\prime}(u)^{2}+\frac{\left(\partial_{1} \psi\right)^{2}}{\psi^{2}\left(\partial_{1} u\right)^{2}} Z(u)^{2}-2 Z(u) Z^{\prime}(u) \frac{\partial \psi}{\psi \partial_{1} u}\right) \\
&+2 \psi Z(u) g_{\varepsilon}^{\prime}(u) w-\psi Z(u) Z^{\prime \prime}(u) w^{2}-\psi w g_{\varepsilon}(u) Z^{\prime}(u) \\
&\left.-2 \psi Z^{\prime}(u) \partial_{1} u \partial_{1} w\right]+w\left(\Delta \psi-2 \frac{|\nabla \psi|^{2}}{\psi}\right) .
\end{aligned}
$$

Let us estimate some of the terms appearing in the above expression. From (28) and (18) we obtain the relation

$$
\psi \partial_{1} w=-w \partial_{1} \psi
$$

and therefore

$$
\begin{aligned}
2 \psi Z^{\prime}(u) \partial_{1} u \partial_{1} w & =-2 Z^{\prime}(u)|\nabla u| w \partial_{1} \psi \\
& \leq 2 Z^{\prime}(u) Z(u)^{1 / 2} \psi^{1 / 2} w^{3 / 2} \sup _{D} \frac{|\nabla \psi|}{\psi^{1 / 2}} .
\end{aligned}
$$

On the other hand,

$$
\begin{aligned}
\frac{1}{2} w^{2} \frac{\left(\partial_{1} \psi\right)^{2}}{\psi\left(\partial_{1} u\right)^{2}} Z(u)^{2} & =\frac{1}{2} \frac{\left(\partial_{1} \psi\right)^{2}}{\psi} Z(u) w \\
& \geq-\frac{1}{2}\left(\sup _{D} \frac{|\nabla \psi|^{2}}{\psi}\right) Z(u) w .
\end{aligned}
$$

We also have

$$
-w^{2} Z(u) Z^{\prime}(u) \frac{\partial_{1} \psi}{\partial_{1} u} \geq-\left(\sup _{D} \frac{|\nabla \psi|}{\psi^{1 / 2}}\right) Z^{\prime}(u) Z(u)^{1 / 2} \psi^{1 / 2} w^{3 / 2} .
$$

The last term to estimate is

$$
w\left(\Delta \psi-2 \frac{|\nabla \psi|^{2}}{\psi}\right) \geq-w \sup _{D}\left(\Delta \psi-2 \frac{|\nabla \psi|^{2}}{\psi}\right) .
$$

Combining (29) with (30)-(33) we obtain at $\left(x_{0}, t_{0}\right)$,

$$
\begin{aligned}
\Delta v-v_{t} \geq \frac{1}{Z(u)}\left[\psi w^{2}\left(\frac{1}{2} Z^{\prime}(u)^{2}-Z(u) Z^{\prime \prime}(u)\right)\right. & \\
+w\left(2 \psi Z(u) g_{\varepsilon}^{\prime}(u)-\psi g_{\varepsilon}(u) Z^{\prime}(u)\right. & -K Z(u)) \\
& \left.-K Z^{\prime}(u) Z(u)^{1 / 2} \psi^{1 / 2} w^{3 / 2}\right],
\end{aligned}
$$

where $K>0$ is a constant.

As we said before, if $v\left(x_{0}, t_{0}\right)$ is large enough, then the right-hand side of (34) must be positive, which would contradict (22). For this purpose we need to establish the following estimates uniformly for all $0<\varepsilon<1$ :

$$
\begin{aligned}
Z^{\prime}(u) Z(u)^{1 / 2} & \leq C\left(\frac{1}{2} Z^{\prime}(u)^{2}-Z^{\prime \prime}(u) Z(u)\right), \\
Z(u)\left|g_{\varepsilon}^{\prime}(u)\right| & \leq C\left(\frac{1}{2} Z^{\prime}(u)^{2}-Z^{\prime \prime}(u) Z(u)\right), \\
Z^{\prime}(u) g_{\varepsilon}(u) & \leq C\left(\frac{1}{2} Z^{\prime}(u)^{2}-Z^{\prime \prime}(u) Z(u)\right), \\
Z(u) & \leq C\left(\frac{1}{2} Z^{\prime}(u)^{2}-Z^{\prime \prime}(u) Z(u)\right),
\end{aligned}
$$


for all $0 \leq u \leq M$ where

$$
M=\left\|u^{\varepsilon}\right\|_{L^{\infty}(\Omega \times(0, T))},
$$

and $C$ depends only on $\beta, M, f$ and $f^{\prime}$.

Suppose for a moment that (35)-(38) have been proved. Then inequality (34) implies that

$$
\begin{aligned}
\Delta v-v_{t} & \geq \frac{\frac{1}{2} Z^{\prime}(u)^{2}-Z^{\prime \prime}(u) Z(u)}{Z(u)}\left(\psi w^{2}-C\left(w+\psi^{1 / 2} w^{3 / 2}\right)\right) \\
& =\frac{\frac{1}{2} Z^{\prime}(u)^{2}-Z^{\prime \prime}(u) Z(u)}{Z(u) \psi}\left(v^{2}-C\left(v+v^{3 / 2}\right)\right) .
\end{aligned}
$$

Thus if $v\left(x_{0}, t_{0}\right)>C_{1}$ for some large $C_{1}$ independent of $\varepsilon$, we obtain a contradiction to (22).

We now turn to the proof of (35)-(38). First note that (17) furnishes

$$
\begin{aligned}
\frac{1}{2} Z^{\prime}(u)^{2}-Z^{\prime \prime}(u) Z(u) & =\frac{1}{2}\left((1-\beta) u^{-\beta}+1\right)^{2}+\beta(1-\beta) u^{-1-\beta}\left(u^{1-\beta}+u\right) \\
& \geq \frac{1}{2}(1-\beta)^{2}\left(u^{-2 \beta}+1\right) .
\end{aligned}
$$

To verify (35) observe that

$$
\begin{aligned}
Z^{\prime}(u) Z(u)^{1 / 2} & \leq C\left(u^{-\beta}+1\right)\left(u^{(1-\beta) / 2}+u^{1 / 2}\right) \\
& \leq C\left(u^{-2 \beta}+1\right)
\end{aligned}
$$

since the exponent $-2 \beta$ is smaller than all others appearing in (40).

Now we proceed with (36). Observe that

$$
g_{\varepsilon}^{\prime}(u)=\frac{\varepsilon-\beta u}{(u+\varepsilon)^{2+\beta}}-f^{\prime}(u) .
$$

Let us first deal with the case $u<\frac{\varepsilon}{\beta}$. Then

$$
\left|g_{\varepsilon}^{\prime}(u)\right| \leq \varepsilon^{-1-\beta}+\left\|f^{\prime}\right\|_{L^{\infty}(0, M)}=\varepsilon^{-1-\beta}+C .
$$

Therefore,

$$
\begin{aligned}
\left|g_{\varepsilon}^{\prime}(u)\right| Z(u) & \leq C\left(\varepsilon^{-1-\beta}+C\right)\left(u^{1-\beta}+u\right) \\
& \leq C\left(\varepsilon^{-2 \beta}+u \varepsilon^{-1-\beta}+u^{1-\beta}+u\right) \\
& \leq C\left(\varepsilon^{-2 \beta}+1\right) \\
& \leq C\left(u^{-2 \beta}+1\right)
\end{aligned}
$$

where $C$ depends on $\beta, M, f$ and $f^{\prime}$.

In the case $u \geq \varepsilon / \beta$ we have

$$
\begin{aligned}
\left|g_{\varepsilon}^{\prime}(u)\right| Z(u) & =\left|\left(\frac{\beta u-\varepsilon}{(u+\varepsilon)^{2+\beta}}+f^{\prime}(u)\right)\left(u^{1-\beta}+u\right)\right| \\
& \leq C\left(u^{-1-\beta}+\left\|f^{\prime}\right\|_{L^{\infty}(0, M)}\right)\left(u^{1-\beta}+u\right) \\
& \leq C\left(u^{-2 \beta}+1\right) .
\end{aligned}
$$

Inequality (37) is verified in the sequel

$$
Z^{\prime}(u) g_{\varepsilon}(u) \leq C\left(u^{-\beta}+1\right) u^{-\beta} \leq C\left(u^{-2 \beta}+1\right) .
$$

Finally (38) follows easily from the definitions. 
Next we present a version of the previous lemma that allows the initial condition to be $u_{0} \in L^{\infty}(\Omega), u_{0} \geq 0$. First we need a technical result.

Lemma 2.3. Let $\varphi \in C^{2}(\Omega) \cap C(\bar{\Omega}), \varphi \geq 0$ with $\varphi=0$ on $\partial \Omega$. Then

$$
\begin{aligned}
|\nabla \varphi(x)|^{2} \leq C( & \left\|D^{2} \varphi\right\|_{L^{\infty}(\Omega)}^{2} \min \left(1,\|D \varphi\|_{L^{\infty}(\Omega)}^{-1}\right) \delta(x) \\
& \left.+\max \left(1,\|D \varphi\|_{L^{\infty}(\Omega)}\right) \delta(x)^{-1}\right) \varphi(x) \quad \forall x \in \Omega,
\end{aligned}
$$

where $C \geq 0$ is a constant depending only on $\Omega$ and $\delta(x)$ is given by (6).

Proof. Pick a point $x \in \Omega$ and suppose $\nabla \varphi(x) \neq 0$. Define the vector $e=$ $\nabla \varphi(x) /|\nabla \varphi(x)|$. Then for $0<t<\delta(x) / 2$ we have

$$
0 \leq \varphi(x-t e)=\varphi(x)-t|\nabla \varphi(x)|+\frac{t^{2}}{2} D^{2} \varphi(\xi) e \cdot e,
$$

where $\xi$ lies in the segment from $x$ to $x-t e$. Therefore,

$$
|\nabla \varphi(x)| \leq \frac{1}{t} \varphi(x)+t\left\|D^{2} \varphi\right\|_{L^{\infty}(\Omega)} .
$$

We choose

$$
t=a \delta(x)^{1 / 2} \varphi(x)^{1 / 2} \min \left(1,\|D \varphi\|_{L^{\infty}(\Omega)}^{-1 / 2}\right),
$$

where $a>0$ depends only on $\Omega$ to be fixed next. Observe that in general

$$
\sup _{x \in \Omega} \frac{\varphi(x)}{\delta(x)} \leq C\|D \varphi\|_{L^{\infty}(\Omega)}
$$

for some constant $C>0$ depending only on $\Omega$. Thus one can choose $a$ depending only on $\Omega$ so that

which implies

$$
a^{2} \sup _{\Omega} \frac{\varphi}{\delta} \min \left(1,\|D \varphi\|_{L^{\infty}(\Omega)}^{-1}\right)<\frac{1}{4}
$$

$$
t<\frac{1}{2} \delta(x)
$$

Our choice of $a$, (42) and (41) yield the desired result.

Lemma 2.4. Let $u_{0} \in L^{\infty}(\Omega)$ with $u_{0} \geq 0$. Let $u^{\varepsilon} \in L^{\infty}(\Omega \times(0, T))$ denote the solution of $\left(P_{\varepsilon}\right]$. Then there exists $C_{2}$ such that

$$
\left|\nabla u^{\varepsilon}(x, t)\right|^{2} \leq C_{2} \psi(x)^{-1}\left(u^{\varepsilon}(x, t)^{1-\beta}+\frac{1}{t} u^{\varepsilon}(x, t)\right) \quad \forall x \in \Omega, t \in(0, T),
$$

where $\psi$ is as in (12), and $C_{2}$ depends only on $T, \Omega, N, \beta, \psi,\left\|u^{\varepsilon}\right\|_{L^{\infty}(\Omega \times(0, T))}, f$, $f^{\prime}$.

Proof. For the sake of notation we write $u=u^{\varepsilon}$. First we consider the case $u_{0} \in$ $C^{2, \mu}(\bar{\Omega}), 0<\mu<1$, and $u_{0}=0$ on $\partial \Omega$. Define

$$
Z(u, t)=u^{1-\beta}+\frac{1}{t} u
$$

and

$$
w=\frac{|\nabla u|^{2}}{Z(u, t)}, \quad v=w \psi
$$

The constant $C_{2}$ will be fixed later. The function $v$ is continuous in $\bar{\Omega} \times[0, T]$ and since $u_{0} \in C^{2, \mu}(\bar{\Omega})$ there is a constant $C$ such that $\|u(\cdot, t)\|_{C^{2, \mu}(\bar{\Omega})} \leq C$ for 
$0 \leq t \leq T$; see [LSU. Notice that this constant may depend on $\varepsilon$ and $u_{0}$. Thus by Lemma 2.3

$$
v(x, t) \leq t \psi \frac{|\nabla u|^{2}}{u} \leq t \psi \frac{C}{\delta} \leq C^{\prime} t,
$$

for some constant $C^{\prime}>0$. Hence, for a sufficiently small $t_{1}>0$ we obtain

$$
v(x, t)<C_{2} \quad \forall x \in \Omega, t \in\left(0, t_{1}\right) .
$$

A contradiction is risen in the same manner as in Lemma 2.1. by assuming that

$$
\sup _{\Omega \times(0, T)} v>C_{2} .
$$

By (44) and since $v$ is continuous on $\bar{\Omega} \times[0, T]$, the supremum in (45) is attained at some point $\left(x_{0}, t_{0}\right) \in \Omega \times\left[t_{1}, T\right]$ and therefore $\nabla v\left(x_{0}, t_{0}\right)=0$ and $\Delta v\left(x_{0}, t_{0}\right)-$ $v_{t}\left(x_{0}, t_{0}\right) \leq 0$.

We have the following expression for $w_{t}$,

$$
w_{t}=\frac{2 \partial_{j} u \partial_{j}\left(u_{t}\right) Z(u)-|\nabla u|^{2} Z_{u}(u) u_{t}-|\nabla u|^{2} Z_{t}(u)}{Z(u)^{2}},
$$

where $Z_{u}$ and $Z_{t}$ denote the partial derivatives of $Z$ with respect to $u$ and $t$. Thus, at $\left(x_{0}, t_{0}\right)$ obtain

$$
\begin{aligned}
& \Delta v-v_{t} \geq \frac{1}{Z(u)}\left[\psi w^{2}\left(\frac{1}{2} Z_{u}(u)^{2}-Z(u) Z_{u u}(u)\right)\right. \\
& +w\left(2 \psi Z(u) g_{\varepsilon}^{\prime}(u)-\psi\right. \\
& \left.\quad g_{\varepsilon}(u) Z_{u}(u)-K Z(u)\right) \\
& \left.\quad-K Z_{u}(u) Z(u)^{1 / 2} \psi^{1 / 2} w^{3 / 2}+Z_{t} \psi w\right],
\end{aligned}
$$

where $K$ is a constant that depends only on $\psi$. We claim that the following inequalities hold

$$
\begin{aligned}
Z_{u} Z^{1 / 2} & \leq C\left(\frac{1}{2} Z_{u}^{2}-Z_{u u} Z\right), \\
Z\left|g_{\varepsilon}^{\prime}\right| & \leq C\left(\frac{1}{2} Z_{u}^{2}-Z_{u u} Z\right), \\
Z_{u} g_{\varepsilon} & \leq C\left(\frac{1}{2} Z_{u}^{2}-Z_{u u} Z\right), \\
Z & \leq C\left(\frac{1}{2} Z_{u}^{2}-Z_{u u} Z\right), \\
\left|Z_{t}\right| & \leq C\left(\frac{1}{2} Z_{u}^{2}-Z_{u u} Z\right),
\end{aligned}
$$

uniformly for all $0<t<T, 0<\varepsilon<1$ and $0<u \leq M$, where $M=\|u\|_{L^{\infty}(\Omega \times(0, T))}$ and $C$ depends only on $T, \beta, M, f, f^{\prime}$. These inequalities together with (46)) produce the contradiction $\Delta v\left(x_{0}, t_{0}\right)-v_{t}\left(x_{0}, t_{0}\right)>0$ if $v\left(x_{0}, t_{0}\right)>C_{2}$.

For the case $u_{0} \in L^{\infty}(\Omega)$ we use an approximation scheme. Let $u_{0}^{j}$ be a sequence in $C^{2, \mu}(\bar{\Omega})$ of initial data with $u_{0}^{j}=0$ on $\partial \Omega$ such that $u_{0}^{j} \geq 0, u_{0}^{j} \rightarrow u_{0}$ a.e. and

$$
\limsup _{j \rightarrow \infty}\left\|u_{0}^{j}\right\|_{L^{\infty}(\Omega)} \leq\left\|u_{0}\right\|_{L^{\infty}(\Omega)} .
$$

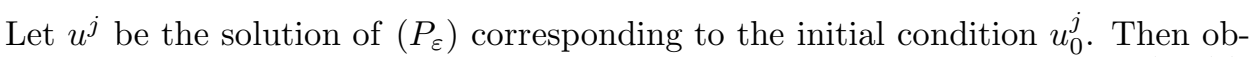
serve that (43) implies that for any $t_{1}>0$ and $\Omega^{\prime} \subset \subset \Omega$ each element $u^{j}(\cdot, t)^{(1+\beta) / 2}$ is uniformly Lipschitz in $\Omega^{\prime}$ for $t \in\left(t_{1}, T\right)$. Thus for a subsequence $j_{k} \nearrow \infty$ the sequence $u^{j}$ converges and the limit is $u$, the solution of $\left[P_{\varepsilon}\right]$. Passing to the limit as $j \rightarrow \infty$ in (43) we get the conclusion. 
A direct consequence of the two previous lemmas is the following.

Corollary 2.5. Under the same hypotheses of Lemma 2.4 we have

$$
\left|\nabla u^{\varepsilon}(x, t)\right|^{2} \leq C_{3} \psi(x)^{-1}\left(u^{\varepsilon}(x, t)^{1-\beta}+\frac{1}{\min (t, 1)} u^{\varepsilon}(x, t)\right) \quad \forall x \in \Omega, t \in(0, T),
$$

where $C_{3}$ is a constant depending only on $\Omega, N, \beta, \psi,\left\|u^{\varepsilon}\right\|_{L^{\infty}(\Omega \times(0, T))}, f, f^{\prime}$ but is independent of $T$.

Proof. We first apply Lemma 2.4 and deduce that

$$
|\nabla u(x, t)|^{2} \leq C_{2} \psi(x)^{-1}\left(u(x, t)^{1-\beta}+\frac{1}{t} u(x, t)\right) \quad \forall x \in \Omega, t \in(0, \min (1, T)) .
$$

To proceed further we assume that $T>1$. The desired conclusion follows by applying Lemma 2.1 with initial condition $u(1)$.

\section{Estimates in time}

In the sequel we establish regularity in time.

Lemma 3.1. Let $u_{0} \in L^{\infty}(\Omega), u_{0} \geq 0$ and let $u^{\varepsilon}$ denote the solution of $\left(P_{\varepsilon}\right)$ with initial condition $u_{0}$. Then for any $\Omega^{\prime} \subset \subset \Omega$ and any $\tau>0$ there exists $C>0$ such that

$$
\left|u_{t}^{\varepsilon}(x, t)\right| \leq C u^{\varepsilon}(x, t)^{-\beta} \quad \forall x \in \Omega^{\prime}, \forall t \in(\tau, T),
$$

where $C$ depends only on $\tau$, $\operatorname{dist}\left(\Omega^{\prime}, \partial \Omega\right),\left\|u^{\varepsilon}\right\|_{L^{\infty}(\Omega \times(0, T))}, f, f^{\prime}, N, \beta$.

If, moreover, $u_{0} \in C^{2}(\Omega), u_{0} \geq 0$, then $u^{\varepsilon}$ satisfies

$$
\left|u_{t}^{\varepsilon}(x, t)\right| \leq C u^{\varepsilon}(x, t)^{-\beta} \quad \forall x \in \Omega^{\prime}, \forall t \in(0, T),
$$

where $\Omega^{\prime} \subset \subset \Omega$.

Proof. We denote $u^{\varepsilon}$ by $u$. We start with the interior estimate, assuming only that $u_{0} \in L^{\infty}(\Omega)$. Fix

$$
r_{0}=\min \left(1, \operatorname{dist}\left(\Omega^{\prime}, \partial \Omega\right), \sqrt{\tau}\right)>0 \quad \text { and } \quad L=\sup _{\bar{\Omega} \times[0, T]} u .
$$

Let $x_{0} \in \Omega^{\prime}, t_{0} \in(\tau, T)$. We consider the rescaled and translated function, respectively by $r>0$ and $x_{0}$, namely

$$
\tilde{u}(x, t)=r^{-\alpha} u\left(r x+x_{0}, r^{2} t+t_{0}\right)
$$

where $\alpha=\frac{2}{1+\beta}$ and which satisfies

$$
\tilde{u}_{t}-\Delta \tilde{u}+r^{2-\alpha} g_{\varepsilon}\left(r^{\alpha} \tilde{u}\right)=0
$$

in the corresponding translated and scaled domain.

We fix

$$
r=\frac{1}{2} r_{0}\left(\frac{u(0,0)}{L}\right)^{1 / \alpha}
$$

so that

$$
\tilde{u}(0,0)=2^{\alpha} r_{0}^{-\alpha} L .
$$

Observe that by the choice of $r_{0}$, the function $\tilde{u}$ is defined in $\bar{B}_{1} \times[-1,0]$, is $C^{1}$ and satisfies (50) in $B_{1} \times(-1,0)$.

Our goal is to prove that

$$
\left|\tilde{u}_{t}(0,0)\right| \leq C_{1} .
$$


We first observe that

$$
|\nabla \tilde{u}|^{2} \leq C \tilde{u}^{1-\beta} \quad \text { in } B_{1} \times(-1,0) .
$$

In fact, by Corollary 2.5 we know that

$$
|\nabla u|^{2} \leq C\left(u^{1-\beta}+u\right) \quad \text { in } B_{r}\left(x_{0}\right) \times\left(t_{0}-r^{2}, t_{0}\right),
$$

so that

$$
\begin{aligned}
|\nabla \tilde{u}|^{2}=r^{2-2 \alpha}|\nabla u|^{2} & \leq C r^{2-2 \alpha}\left(u^{1-\beta}+u\right) \\
& \leq C\left(\tilde{u}^{1-\beta}+r^{2-\alpha} \tilde{u}\right) \\
& \leq C \tilde{u}^{1-\beta} .
\end{aligned}
$$

We also remark that from (53) we get

$$
\left|\nabla \tilde{u}^{(1+\beta) / 2}\right| \leq C \quad \text { in } B_{1} \times(-1,0) .
$$

Let $\psi$ be a smooth function on $\bar{B}_{1}$ such that $\psi>0$ in $B_{1}, \psi=0$ on $\partial B_{1}$ and $|\nabla \psi|^{2} / \psi$ is bounded in $B_{1}$, thus $\psi$ is the function defined in the beginning of this section with $B_{1}$ instead of $D$.

We multiply equation (50) by $\tilde{u}_{t} \psi$ and integrate over $B_{1}$,

$$
\int_{B_{1}}\left(\tilde{u}_{t}\right)^{2} \psi=-\frac{1}{2} \frac{d}{d t} \int_{B_{1}}|\nabla \tilde{u}|^{2} \psi-\int_{B_{1}} \tilde{u}_{t} \nabla \tilde{u} \nabla \psi-r^{2-2 \alpha} \frac{d}{d t} \int_{B_{1}} G_{\varepsilon}\left(r^{\alpha} \tilde{u}\right) \psi,
$$

where

Hence

$$
G_{\varepsilon}(u)=\int_{0}^{u} g_{\varepsilon}(s) d s
$$

$$
\begin{aligned}
\int_{B_{1}}\left(\tilde{u}_{t}\right)^{2} \psi \leq & -\frac{1}{2} \frac{d}{d t} \int_{B_{1}}|\nabla \tilde{u}|^{2} \psi+\frac{1}{2} \int_{B_{1}}\left(\tilde{u}_{t}\right)^{2} \psi+C\left(\sup _{B_{1}} \frac{|\nabla \psi|^{2}}{\psi}\right) \int_{B_{1}}|\nabla \tilde{u}|^{2} \\
& -r^{2-2 \alpha} \frac{d}{d t} \int_{B_{1}} G_{\varepsilon}\left(r^{\alpha} \tilde{u}\right) \psi
\end{aligned}
$$

and therefore

$$
\frac{1}{2} \int_{B_{1}}\left(\tilde{u}_{t}\right)^{2} \psi \leq-\frac{1}{2} \frac{d}{d t} \int_{B_{1}}|\nabla \tilde{u}|^{2} \psi+C \int_{B_{1}}|\nabla \tilde{u}|^{2}-r^{2-2 \alpha} \frac{d}{d t} \int_{B_{1}} G_{\varepsilon}\left(r^{\alpha} \tilde{u}\right) \psi .
$$

Integrating from $t_{1} \in(-1,0)$ to 0 we obtain

$$
\begin{aligned}
& \int_{t_{1}}^{0} \int_{B_{1}}\left(\tilde{u}_{t}\right)^{2} \psi d x d t \\
& \quad \leq \int_{B_{1}}\left|\nabla \tilde{u}\left(t_{1}\right)\right|^{2}-|\nabla \tilde{u}(0)|^{2} d x+C \int_{t_{1}}^{0} \int_{B_{1}}|\nabla \tilde{u}|^{2} d x d t \\
& \quad \quad+2 r^{2-2 \alpha} \int_{B_{1}} G_{\varepsilon}\left(r^{\alpha} \tilde{u}\left(t_{1}\right)\right) \psi d x-2 r^{2-2 \alpha} \int_{B_{1}} G_{\varepsilon}\left(r^{\alpha} \tilde{u}(0)\right) \psi d x .
\end{aligned}
$$

Recall that

$$
g_{\varepsilon}(u)=\frac{u}{(u+\varepsilon)^{1+\beta}}-f(u)
$$

and $f(u) \geq 0$ hence

$$
-F(u) \leq G_{\varepsilon}(u) \leq \frac{u^{1-\beta}}{1-\beta}
$$


where $F(u)=\int_{0}^{u} f(s) d s$. Since $F(u) \leq C u(1+u)$ and $r \leq 1$, we have for all $\tilde{u} \geq 0$,

$$
\begin{aligned}
r^{2-2 \alpha} F\left(r^{\alpha} \tilde{u}\right) & \leq C r^{2-\alpha} \tilde{u}\left(1+r^{\alpha} \tilde{u}\right) \\
& \leq C\left(1+\tilde{u}^{2}\right) .
\end{aligned}
$$

Consequently,

$$
-C\left(1+\tilde{u}^{2}\right) \leq r^{2-2 \alpha} G_{\varepsilon}\left(r^{\alpha} \tilde{u}\right) \leq \frac{\tilde{u}^{1-\beta}}{1-\beta} .
$$

Using the above inequality together with (53) we deduce from (55) that (56)

$$
\int_{t_{1}}^{0} \int_{B_{1}}\left(\tilde{u}_{t}\right)^{2} \psi \leq C\left[1+\int_{B_{1}} \tilde{u}\left(t_{1}\right)^{1-\beta} d x+\int_{t_{1}}^{0} \int_{B_{1}} \tilde{u}^{1-\beta} d x d t+\int_{B_{1}} \tilde{u}(0)^{2} d x\right] .
$$

Now we need an estimate for $\tilde{u}$ in terms of the left-hand side of (56). Take $t \in(-1 / 2,0)$. For $x \in B_{1}$,

$$
\tilde{u}(x, 0)-\tilde{u}(x, t)=\int_{t}^{0} \tilde{u}_{t}(x, s) d s .
$$

Integrating over $B_{r_{1}}$, where $r_{1}=|t|^{1 /(3 N)}$, we have for some $\bar{x} \in B_{r_{1}}$

$$
\tilde{u}(\bar{x}, 0)-\tilde{u}(\bar{x}, t)=\frac{C}{r_{1}^{N}} \int_{t}^{0} \int_{B_{r_{1}}} \tilde{u}_{t}
$$

and by Schwarz's inequality

$$
(\tilde{u}(\bar{x}, 0)-\tilde{u}(\bar{x}, t))^{2} \leq \frac{C|t|}{r_{1}^{N}} \int_{t}^{0} \int_{B_{r_{1}}}\left(\tilde{u}_{t}\right)^{2},
$$

taking $r_{1}=|t|^{1 /(3 N)}$ we obtain

$$
|\tilde{u}(\bar{x}, t)-\tilde{u}(\bar{x}, 0)| \leq C|t|^{1 / 3}\left(\int_{t}^{0} \int_{B_{1}}\left(\tilde{u}_{t}\right)^{2} \psi\right)^{1 / 2} .
$$

Here we have used that $\psi$ has a positive lower bound on the ball $B_{r_{1}}$ for $r_{1} \leq$ $(1 / 2)^{1 /(3 N)}<1$.

On the other hand, by (54) for any $y \in B_{1}$ and $t \in(-1 / 2,0)$,

$$
\tilde{u}(y, t) \leq C\left(|y-\bar{x}|^{2 /(1+\beta)}+\tilde{u}(\bar{x}, t)\right) \leq C(1+\tilde{u}(\bar{x}, t)) .
$$

Hence, combining this with (57) we obtain

$$
\tilde{u}(y, t) \leq C\left(1+|t|^{1 / 3}\left(\int_{t}^{0} \int_{B_{1}}\left(\tilde{u}_{t}\right)^{2} \psi\right)^{1 / 2}\right), \quad \forall y \in B_{1}, t \in(-1 / 2,0) .
$$

In inequality (56) we now take $t_{1} \in(-1 / 2,0)$ and use (58) to get

$$
\begin{aligned}
\int_{t_{1}}^{0} \int_{B_{1}}\left(\tilde{u}_{t}\right)^{2} \psi \leq C[1 & +\left|t_{1}\right|^{(1-\beta) / 3}\left(\int_{t_{1}}^{0} \int_{B_{1}}\left(\tilde{u}_{t}\right)^{2} \psi\right)^{(1-\beta) / 2} \\
& \left.+\int_{t_{1}}^{0} \int_{B_{1}}|t|^{(1-\beta) / 3}\left(\int_{t}^{0} \int_{B_{1}}\left(\tilde{u}_{t}\right)^{2} \psi\right)^{(1-\beta) / 2} d x d t\right] \\
\leq C & {\left[1+\left|t_{1}\right|^{(1-\beta) / 3} \int_{t_{1}}^{0} \int_{B_{1}}\left(\tilde{u}_{t}\right)^{2} \psi\right] }
\end{aligned}
$$


by Young's inequality. By choosing $\sigma>0$ small we see that for $t_{1} \in(-\sigma, 0)$ there is a constant $C$ independent of $t_{1}$ such that

$$
\int_{t_{1}}^{0} \int_{B_{1}}\left(\tilde{u}_{t}\right)^{2} \psi \leq C
$$

Now, using (57) combined with (52) and (54) we conclude that there is $\rho>0$ small such that $\tilde{u}(x, t) \in\left[C_{0} / 2,2 C_{0}\right]$ where $C_{0}=2{ }^{\alpha} r_{0}^{-\alpha} L$ for all $x \in B_{\rho}$ and $t \in(-\rho, 0)$. Thus

$$
\left|\tilde{u}_{t}-\Delta \tilde{u}\right| \leq C \quad \text { in } B_{\rho} \times(-\rho, 0)
$$

and

$$
|\tilde{u}| \leq C \quad \text { on } B_{\rho} \times\{-\rho\} \text { and } \partial B_{\rho} \times(-\rho, 0)
$$

together with standard parabolic estimates [LSU] imply that

$$
\left|\tilde{u}_{t}(0,0)\right| \leq C_{1} .
$$

It remains to treat the case $u_{0} \in C^{2}(\Omega)$ and show that $u$ satisfies (47). The argument is similar to the above one, except for the fact that now we use

$$
r_{0}=\min \left(1, \operatorname{dist}\left(\Omega^{\prime}, \partial \Omega\right)\right) / 4>0
$$

in our rescaling procedure. Let $r$ be given by formula (51) with this new definition of $r_{0}$. Let $x_{0} \in \Omega^{\prime}$ and $t_{0}>0$ and define $\tilde{u}$ as in (49), which now satisfies the rescaled equation (67) in $B_{1} \times\left(-t_{0} / r^{2}, 0\right)$.

Since $u_{0} \in C^{2}(\Omega)$ applying Lemma 2.3 to $u_{0}$ multiplied by a suitable cutoff function we see that

$$
\sup _{D} \frac{\left|\nabla u_{0}\right|^{2}}{u_{0}}<\infty
$$

for any $D \subset \subset \Omega$.

Let $D \subset \subset \Omega$ be a smooth subdomain such that $B_{r_{0}}\left(x_{0}\right) \subset D$. Then using Lemma 2.1 in the domain $D$ instead of $\Omega$ we conclude that

$$
|\nabla u|^{2} \leq C u^{1-\beta} \quad \text { in } B_{r_{0}}\left(x_{0}\right) \times[0, T] .
$$

This implies

$$
|\nabla \tilde{u}|^{2} \leq C \tilde{u}^{1-\beta} \quad \text { in } B_{1} \times\left(-t_{0} / r^{2}, 0\right) .
$$

The calculations we performed before still apply if we restrict $t_{1}$. Thus, taking $t_{1} \in\left(-t_{0} / r^{2}, 0\right)$, there is $\rho>0$ sufficiently small and independent of $\varepsilon$ such that

$$
\left|\tilde{u}_{t}-\Delta \tilde{u}\right| \leq C \quad \text { in } B_{\rho} \times\left(-\min \left(\rho, t_{0} / r^{2}\right), 0\right)
$$

and

$$
|\tilde{u}| \leq C \quad \text { on } \partial B_{\rho} \times\left(-\min \left(\rho, t_{0} / r^{2}\right), 0\right) .
$$

We shall consider two cases: if $\rho<t_{0} / r^{2}$, then arguing as above, with the aid of (58), we deduce that $|\tilde{u}(-\rho)| \leq C$ in $B_{\rho}$. By standard parabolic estimates [LSU], we deduce (59), because $\tilde{u}$ satisfies a parabolic equation where the initial condition $\tilde{u}(-\rho)$ is given at a fixed time separation from 0 and is bounded in $B_{\rho}$. In the second alternative, $\rho \geq t_{0} / r^{2}$, we see that we still obtain (59) since, even though the initial condition $\tilde{u}\left(-t_{0} / r^{2}\right)$ is specified at a time close to 0 , we can estimate the Hölder semi-norm

$$
\left[\tilde{u}\left(-t_{0} / r^{2}\right)\right]_{C^{1, \mu}\left(B_{\rho}\right)}=\sup _{x, y \in B_{\rho}} \frac{\left|\tilde{u}\left(x,-t_{0} / r^{2}\right)-\tilde{u}\left(y,-t_{0} / r^{2}\right)\right|}{|x-y|^{\mu}} \leq C,
$$


where $C$ is independent of $r$ if $\mu \geq \alpha-1$ (note that $0<\alpha-1<1$ ). Since we also know that $\left|\tilde{u}\left(-t_{0} / r^{2}\right)\right| \leq C$ in $B_{\rho}$, by standard Hölder estimates for the heat equation with initial condition in $C^{1, \mu}\left(\bar{B}_{\rho}\right), \alpha-1 \leq \mu<1$, we deduce (59).

Combining Corollary 2.5 and Lemma 3.1 we deduce

Corollary 3.2. If $u_{0} \in L^{\infty}(\Omega), u_{0} \geq 0$, then for any $\tau>0$ and $\Omega^{\prime} \subset \subset \Omega$ there exists $C$ such that

$$
\left|u^{\varepsilon}(x, t)-u^{\varepsilon}(x, s)\right| \leq C|t-s|^{1 /(1+\beta)} \quad \forall x \in \Omega^{\prime}, \forall t, s \in(\tau, T)
$$

and

$$
\left|\nabla u^{\varepsilon}(x, t)-\nabla u^{\varepsilon}(y, t)\right| \leq C|x-y|^{\frac{1-\beta}{1+\beta}} \quad \forall x, y \in \Omega^{\prime}, \forall t \in(\tau, T) .
$$

If, moreover, $u_{0} \in C^{2}(\Omega)$, then one can replace $(\tau, T)$ with $[0, T)$ in the estimates above. The constant $C$ depends only on $\Omega, N, \beta,\left\|u^{\varepsilon}\right\|_{L^{\infty}(\Omega \times(0, T))}, f, f^{\prime}$.

Proof. The Hölder estimate in time follows immediately from Lemma 3.1 ,

Let $\tau>0$ and $\Omega^{\prime} \subset \subset \Omega$ and let $u$ denote $u^{\varepsilon}$. Fix $x_{0}, y_{0} \in \Omega^{\prime}$ and $t_{0}>\tau$. We consider now the following cases.

Case a) Suppose that

$$
\left|x_{0}-y_{0}\right| \leq \theta \max \left(u\left(x_{0}, t_{0}\right)^{1 / \alpha}, u\left(y_{0}, t_{0}\right)^{1 / \alpha}\right),
$$

where $\theta>0$ will be fixed independently of $\varepsilon$. Then, without loss of generality, we assume that

$$
\left|x_{0}-y_{0}\right| \leq \theta u\left(x_{0}, t_{0}\right)^{1 / \alpha} .
$$

Define $r_{0}, r$ and $\tilde{u}$ as in Lemma 3.1 (see (48), (49) and (51) ). It was shown in that lemma that

and

$$
\left|\tilde{u}_{t}-\Delta \tilde{u}\right| \leq C \quad \text { in } B_{\rho} \times(-\rho, 0)
$$

$$
|\tilde{u}| \leq C \quad \text { on } B_{\rho} \times\{-\rho\} \text { and } \partial B_{\rho} \times(-\rho, 0),
$$

where $C$ and $\rho>0$ are independent of $\varepsilon$. By parabolic estimates [LSU] we deduce that $\nabla \tilde{u}$ is $C^{\mu}$ in the variable $x$ for any $0<\mu<1$ in $B_{\rho / 2} \times(-\rho / 2,0)$. Now we fix $\theta=\frac{1}{4} \rho r_{0} L^{-1 / \alpha}$ where $L=\sup _{\bar{\Omega} \times[0, T]} u$. This ensures that if (62) holds, then $\left(y_{0}-x_{0}\right) / r \in B_{\rho / 2}$. Thus, using $\mu=\frac{1-\beta}{1+\beta}$ we get

$$
\left|\nabla \tilde{u}(0,0)-\nabla \tilde{u}\left(\left(y_{0}-x_{0}\right) / r, 0\right)\right| \leq C\left(\left|y_{0}-x_{0}\right| / r\right)^{\mu},
$$

and using the definition of $\tilde{u}$

$$
r^{1-\alpha}\left|\nabla u\left(x_{0}, t_{0}\right)-\nabla u\left(y_{0}, t_{0}\right)\right| \leq C r^{-\mu}\left|y_{0}-x_{0}\right|^{\mu} .
$$

Recalling that $\alpha-1=\frac{1-\beta}{1+\beta}=\mu$ we obtain the estimate

$$
\left|\nabla u\left(x_{0}, t_{0}\right)-\nabla u\left(y_{0}, t_{0}\right)\right| \leq C\left|y_{0}-x_{0}\right|^{\frac{1-\beta}{1+\beta}} .
$$

Case b) If (61) fails, then

$$
\begin{aligned}
\left|\nabla u\left(x_{0}, t_{0}\right)-\nabla u\left(y_{0}, t_{0}\right)\right| & \leq\left|\nabla u\left(x_{0}, t_{0}\right)\right|+\left|\nabla u\left(y_{0}, t_{0}\right)\right| \\
& \leq C\left(u\left(x_{0}, t_{0}\right)^{(1-\beta) / 2}+u\left(y_{0}, t_{0}\right)^{(1-\beta) / 2}\right) \\
& \leq C\left|x_{0}-y_{0}\right|^{\frac{1-\beta}{1+\beta}},
\end{aligned}
$$

where we have used the estimate $|\nabla u| \leq C u^{(1-\beta) / 2}$ which follows from Corollary 2.5 


\section{Existence of A SOLUtion}

In this section we justify the passage to the limit $u^{\varepsilon} \rightarrow u$ as $\varepsilon \rightarrow 0$, proving Theorem 1.1. Throughout this section we assume that $T$ is finite. The existence of a solution defined for all $t$ is obtained by applying Theorem 1.1 for all $T>0$ and then letting $T \rightarrow \infty$.

We also provide arguments for the claims presented in Remark 1.2 .

Step 1. First let us observe that to apply Corollary 3.2 and get estimates that are independent of $\varepsilon$ it is first necessary to show that

$$
\sup _{\varepsilon>0} \max _{\bar{\Omega} \times[0, T]} u^{\varepsilon}<\infty .
$$

This follows by observing that the solution $\bar{u}$ to

$$
\left\{\begin{aligned}
\bar{u}_{t}-\Delta \bar{u} & =f(\bar{u}) & & \text { in } \Omega \times(0, T), \\
\bar{u} & =0 & & \text { on } \partial \Omega \times(0, T), \\
\bar{u}(x, 0) & =u_{0}(x) & & x \in \Omega
\end{aligned}\right.
$$

is a supersolution of $\left(P_{\varepsilon}\right)$ for any $\varepsilon>0$.

Therefore by Corollary $3.2 u=\lim _{\varepsilon \rightarrow 0} u^{\varepsilon}$ exists and is $C^{1 /(1+\beta)}$ in time and $C^{1, \frac{1-\beta}{1+\beta}}$ in space on any $\Omega^{\prime} \times(\tau, T)$ such that $\Omega^{\prime} \subset \subset \Omega$ and $\tau>0$.

Step 2. Let us show that

$$
\chi_{\{u>0\}} g(u) \in L^{1}(\Omega \times[0, T]) .
$$

Observe first that by the previous step $f\left(u^{\varepsilon}\right)$ remains bounded in $L^{\infty}(\Omega \times(0, T))$, thus it suffices to verify that

$$
\chi_{\{u>0\}} u^{-\beta} \in L^{1}(\Omega \times[0, T]) .
$$

Recall that $u^{\varepsilon}$ satisfies

$$
u_{t}^{\varepsilon}-\Delta u_{\varepsilon}+g_{\varepsilon}\left(u^{\varepsilon}\right)=0 \quad \text { in } \Omega \times(0, T) .
$$

Integrating over $\Omega \times(0, T)$ and using the definition of $g_{\varepsilon}($ (5) we see that

$$
0=\int_{\Omega} u^{\varepsilon}(T)-u_{0}+\int_{0}^{T} \int_{\partial \Omega} \frac{\partial u^{\varepsilon}}{\partial n}+\int_{0}^{T} \int_{\Omega} \frac{u^{\varepsilon}}{\left(u^{\varepsilon}+\varepsilon\right)^{1+\beta}}-f\left(u^{\varepsilon}\right)
$$

where $n$ is the unit exterior normal vector to the boundary. But $\frac{\partial u^{\varepsilon}}{\partial n} \leq 0$ and hence

$$
\int_{0}^{T} \int_{\Omega} \frac{u^{\varepsilon}}{\left(u^{\varepsilon}+\varepsilon\right)^{1+\beta}} \leq \int_{\Omega} u_{0}+\int_{0}^{T} \int_{\Omega} f\left(u^{\varepsilon}\right) \leq C
$$

with $C$ independent of $\varepsilon$. Using Fatou's lemma we conclude that (63) holds.

Step 3. If $u_{0} \in C(\Omega), u_{0} \geq 0$, then $u$ is continuous in $\Omega \times[0, T]$. The proof of this claim follows the same steps as in the proof of Theorem 1 in $[\mathrm{P}]$, approximating $u_{0}$ from above and from below by initial conditions in $C^{2}(\Omega)$.

Step 4. To show that $u$ is a solution of $(P)$ in the sense of definition (3)-(4) it is enough to verify that for all $\varphi \in C_{0}^{\infty}(\Omega \times[0, T)$ ) (that is, $\varphi$ is smooth and vanishes away from $T$ and away from $\partial \Omega$ ) we have

$$
-\int_{\Omega} u_{0} \varphi+\int_{0}^{T} \int_{\Omega} \nabla u \nabla \varphi-u \varphi_{t}+\chi_{\{u>0\}} g(u)=0 .
$$


For this observe that $|\nabla u| \in L^{2}(\Omega \times(0, T))$. Indeed, multiplying equation (64) by $u^{\varepsilon}$ and integrating in $\Omega \times(0, T)$ we deduce that

$$
\int_{0}^{T} \int_{\Omega}\left|\nabla u^{\varepsilon}\right|^{2}=-\int_{0}^{T} \int_{\Omega} g_{\varepsilon}\left(u^{\varepsilon}\right) u^{\varepsilon}+\frac{1}{2} \int_{\Omega} u_{0}^{2}-u^{\varepsilon}(T)^{2} \leq C
$$

with $C$ independent of $\varepsilon$. Therefore (65) makes sense and clearly implies (4).

Step 5. If $u_{0} \in C(\Omega), u_{0} \geq 0$, then $u$ is a solution of $(\underline{P})$. Let $\varphi \in C_{0}^{\infty}(\Omega \times[0, T))$ and let $\eta: \mathbb{R} \rightarrow \mathbb{R}$ be a smooth function such that $0 \leq \eta(s) \leq 1$ for all $s, \eta(s)=0$ for $s \leq 1 / 2$ and $\eta(s)=1$ for $s \geq 1$. For $m>0$ we multiply equation (64) by $\varphi \eta\left(u^{\varepsilon} / m\right)$ and integrate on $\Omega \times(\tau, T)$ where $\tau>0$,

$$
\int_{\tau}^{T} \int_{\Omega}\left(u_{t}^{\varepsilon}-\Delta u^{\varepsilon}+g_{\varepsilon}\left(u^{\varepsilon}\right)\right) \varphi \eta\left(u^{\varepsilon} / m\right)=0
$$

Note that

$$
\int_{\tau}^{T} \int_{\Omega} g_{\varepsilon}\left(u^{\varepsilon}\right) \varphi \eta\left(u^{\varepsilon} / m\right)=\int_{\tau}^{T} \int_{\Omega} \chi_{\{u>0\}} g_{\varepsilon}\left(u^{\varepsilon}\right) \varphi \eta\left(u^{\varepsilon} / m\right)
$$

if $m>0$ is fixed and $\varepsilon>0$ is small enough. Therefore, by Step 2 we can apply dominated convergence and conclude that

$$
\lim _{m \rightarrow 0} \lim _{\varepsilon \searrow 0} \int_{\tau}^{T} \int_{\Omega} g_{\varepsilon}\left(u^{\varepsilon}\right) \varphi \eta\left(u^{\varepsilon} / m\right)=\int_{\tau}^{T} \int_{\Omega} \chi_{\{u>0\}} g(u) \varphi .
$$

Let us consider the first term in (66)

$$
\begin{aligned}
\int_{\tau}^{T} \int_{\Omega} u_{t}^{\varepsilon} \varphi \eta\left(u^{\varepsilon} / m\right)=- & \int_{\Omega} u^{\varepsilon}(\tau) \varphi(\tau) \eta\left(u^{\varepsilon}(\tau) / m\right)-\int_{\tau}^{T} \int_{\Omega} u^{\varepsilon} \varphi_{t} \eta\left(u^{\varepsilon} / m\right) \\
& -\frac{1}{m} \int_{\tau}^{T} \int_{\Omega} u^{\varepsilon} \varphi \eta^{\prime}\left(u^{\varepsilon} / m\right) u_{t}^{\varepsilon}
\end{aligned}
$$

There is no problem in passing to the limit with the first two terms on the right-hand side of the above inequality. On the other hand, we claim that

$$
\lim _{m \rightarrow 0} \limsup _{\varepsilon \rightarrow 0}\left|\frac{1}{m} \int_{\tau}^{T} \int_{\Omega} u^{\varepsilon} \varphi \eta^{\prime}\left(u^{\varepsilon} / m\right) u_{t}^{\varepsilon}\right|=0 .
$$

In fact, let $\Omega^{\prime} \subset \subset \Omega$ be such that $\varphi=0$ in $\Omega \backslash \Omega^{\prime} \times(0, T)$. Then by Lemma 3.1

$$
\begin{aligned}
\frac{1}{m}\left|\int_{\tau}^{T} \int_{\Omega} u^{\varepsilon} \varphi \eta^{\prime}\left(u^{\varepsilon} / m\right) u_{t}^{\varepsilon}\right| & \leq \frac{C}{m} \sup |\varphi| \sup \left|\eta^{\prime}\right| \int_{\tau}^{T} \int_{\Omega^{\prime}} \chi_{\left\{0<u^{\varepsilon} \leq m\right\}} u^{\varepsilon}\left|u_{t}^{\varepsilon}\right| \\
& \leq \frac{C}{m} \int_{\tau}^{T} \int_{\Omega^{\prime}} \chi_{\left\{0<u^{\varepsilon} \leq m\right\}}\left(u^{\varepsilon}\right)^{1-\beta} .
\end{aligned}
$$

Hence, letting $\varepsilon \rightarrow 0$,

$$
\begin{aligned}
\limsup _{\varepsilon \rightarrow 0} \frac{1}{m}\left|\int_{\tau}^{T} \int_{\Omega} u^{\varepsilon} \varphi \eta^{\prime}\left(u^{\varepsilon} / m\right) u_{t}^{\varepsilon}\right| & \leq \frac{C}{m} \int_{\tau}^{T} \int_{\Omega^{\prime}} \chi_{\{0<u \leq m\}} u^{1-\beta} \\
& \leq C \int_{\tau}^{T} \int_{\Omega^{\prime}} \chi_{\{0<u \leq m\}} u^{-\beta}
\end{aligned}
$$

Since $u^{-\beta}$ is integrable, by dominated convergence the right-hand side of the inequality above tends to zero as $m \rightarrow 0$. 
Next we consider the term

$$
-\int_{\tau}^{T} \int_{\Omega} \Delta u^{\varepsilon} \varphi \eta\left(u^{\varepsilon} / m\right)=\int_{\tau}^{T} \int_{\Omega} \eta\left(u^{\varepsilon} / m\right) \nabla u^{\varepsilon} \nabla \varphi+\frac{1}{m} \int_{\tau}^{T} \int_{\Omega} \varphi\left|\nabla u^{\varepsilon}\right|^{2} \eta^{\prime}\left(u^{\varepsilon} / m\right) .
$$

Again, the first term on the right-hand side above behaves well as $\varepsilon \rightarrow 0$ and then $m \rightarrow 0$, while for the second we claim that

$$
\left.\lim _{m \rightarrow 0} \limsup _{\varepsilon \rightarrow 0}\left|\frac{1}{m} \int_{\tau}^{T} \int_{\Omega} \varphi\right| \nabla u^{\varepsilon}\right|^{2} \eta^{\prime}\left(u^{\varepsilon} / m\right) \mid=0 .
$$

To prove this we apply Corollary 2.5

$$
\left.\frac{1}{m}\left|\int_{\tau}^{T} \int_{\Omega} \varphi\right| \nabla u^{\varepsilon}\right|^{2} \eta^{\prime}\left(u^{\varepsilon} / m\right) \mid \leq C \int_{\tau}^{T} \int_{\Omega^{\prime}} \chi_{\left\{0<u^{\varepsilon} \leq m\right\}}\left(u^{\varepsilon}\right)^{1-\beta}
$$

and therefore

$$
\limsup _{\varepsilon \rightarrow 0} \frac{1}{m}\left|\int_{\tau}^{T} \int_{\Omega} \varphi\right| \nabla u^{\varepsilon}\left|{ }^{2} \eta^{\prime}\left(u^{\varepsilon} / m\right)\right| \leq C \int_{\tau}^{T} \int_{\Omega^{\prime}} \chi_{\{0<u \leq m\}} u^{-\beta},
$$

which again tends to zero when $m \rightarrow 0$.

Therefore, taking $\varepsilon \rightarrow 0$ and then $m \rightarrow 0$ in (66), and using (67) and (68) we get

$$
-\int_{\Omega} u(\tau) \varphi+\int_{\tau}^{T} \int_{\Omega} \nabla u \nabla \varphi-u \varphi_{t}+\chi_{\{u>0\}} g(u)=0 .
$$

Finally we let $\tau \rightarrow 0$ and use dominated convergence (we know that $\chi_{\{u>0\}} g(u) \in$ $\left.L^{1}(\Omega \times(0, T))\right)$ and the fact that $u$ is continuous at $t=0$ to prove that (65) holds.

Proof of Remark 1.2. We will finish this section by proving the claims made in Remark 1.2, which we recall: if one of the following conditions hold,

i) $u_{0}$ satisfies $u_{0} \geq c \delta^{\nu}$ for some $c>0$ and $1<\nu<\frac{2}{1+\beta}$, or

ii) there exists a sequence $\left(u_{0}^{j}\right)$ in $C(\Omega)$ such that $0 \leq u_{0}^{j} \leq u_{0}$ and $u_{0}^{j} \rightarrow u_{0}$ a.e.,

then the limit $u=\lim _{\varepsilon \rightarrow 0} u^{\varepsilon}$ is a solution of (P), that is, it satisfies (3)-(4).

In order to accomplish this we first observe that the proof of Step 5 above immediately yields

$$
\int_{\Omega} u(\tau) \varphi(\tau) d x+\int_{\tau}^{T} \int_{\Omega}\left(\varphi_{t}+\Delta \varphi\right) u-\chi_{\{u>0\}} g(u) \varphi d x d t=0
$$

for every $\tau>0$ and all $\varphi \in C^{2}(\bar{\Omega} \times[0, T])$ with $\varphi=0$ on $\partial \Omega \times(0, T)$ and $\varphi(T)=0$ in $\Omega$ as claimed in Remark 1.2.

Let $\varphi \in C_{0}^{\infty}(\Omega)$ and let $0<\tau<T$. Then multiplying $(\underline{P})$ by $\varphi$ and integrating by parts we have

$$
\int_{\Omega} u(\tau) \varphi d x=\int_{\Omega} u(T) \varphi d x-\int_{\tau}^{T} \int_{\Omega} \Delta \varphi u-\chi_{\{u>0\}} g(u) \varphi d x d t .
$$

Since $\chi_{\{u>0\}} g(u) \in L^{1}(\Omega)$ we can take $\tau \searrow 0$ above and we conclude that $u(\tau)$ is weakly convergent in $L^{p}(\Omega)$ for any $1<p<\infty$ (recall that $u(\tau)$ is bounded in $\left.L^{\infty}(\Omega)\right)$. Let us write the weak limit as $\hat{u}_{0}$, that is, $u(\tau) \rightarrow \hat{u}_{0}$ weakly in $L^{p}(\Omega)$, where $\hat{u}_{0} \in L^{\infty}(\Omega), \hat{u}_{0} \geq 0$. 
Now let $\varphi \in C^{2}(\bar{\Omega} \times[0, T])$ with $\varphi=0$ on $\partial \Omega \times(0, T)$ and $\varphi(T)=0$ in $\Omega$, and take $\tau \searrow 0$ in (69). We conclude that

$$
\int_{\Omega} \hat{u}_{0} \varphi(0) d x+\int_{0}^{T} \int_{\Omega}\left(\varphi_{t}+\Delta \varphi\right) u-\chi_{\{u>0\}} g(u) \varphi d x d t=0 .
$$

This shows that $u$ satisfies a problem similar to $(P)$, where the only difference is that the initial condition is now $\hat{u}_{0}$ instead of $u_{0}$.

We claim that

$$
\hat{u}_{0} \leq u_{0} \quad \text { a.e. in } \Omega .
$$

Indeed, let $\varphi \in C_{0}^{\infty}(\Omega), \varphi \geq 0$. On one hand we have, by letting $\tau \searrow 0$ in (71),

$$
\int_{\Omega} \hat{u}_{0} \varphi d x=\int_{\Omega} u(T) \varphi d x-\int_{0}^{T} \int_{\Omega} \Delta \varphi u-\chi_{\{u>0\}} g(u) \varphi d x d t .
$$

On the other hand, multiplying $\left[P_{\varepsilon}\right.$ by $\varphi$ and integrating over $[0, T]$ we get

$$
\int_{\Omega} u_{0} \varphi d x=\int_{\Omega} u^{\varepsilon}(T) \varphi d x-\int_{0}^{T} \int_{\Omega} \Delta \varphi u^{\varepsilon}-g_{\varepsilon}\left(u^{\varepsilon}\right) \varphi d x d t .
$$

By Fatou's lemma we deduce

$$
\int_{\Omega} u_{0} \varphi d x \geq \int_{\Omega} u(T) \varphi d x-\int_{0}^{T} \int_{\Omega} \Delta \varphi u-\chi_{\{u>0\}} g(u) \varphi d x d t .
$$

Combining (73) and (74) we conclude (72).

Let us proceed first assuming that i) holds. Then by Lemma 1.9 we see that there are $c^{\prime}>0$ and $\bar{t}>0$ independent of $\varepsilon$ such that

$$
u^{\varepsilon}(t) \geq c^{\prime} \delta^{\nu} \quad \forall t \in(0, \bar{t})
$$

Let $\varphi \in C^{\infty}(\bar{\Omega} \times[0, T])$ with $\varphi=0$ on $\Omega \times\{T\} \cup \partial \Omega \times[0, T]$ and let $0<\tau<\bar{t}$. Then integrating $\left(P_{\varepsilon}\right]$ in $\Omega \times[0, \tau]$ we find

$$
\int_{\Omega} u_{0} \varphi(0) d x+\int_{0}^{\tau} \int_{\Omega}\left(\varphi_{t}+\Delta \varphi\right) u^{\varepsilon}-g_{\varepsilon}\left(u^{\varepsilon}\right) \varphi d x d t=0 .
$$

Thanks to (75) we can pass to the limit as $\varepsilon \rightarrow 0$ in the previous relation. This shows that $u_{0}=\hat{u}_{0}$ in this case and therefore $u$ is a solution of (P).

Now suppose that ii) holds, that is, there is a sequence $\left(u_{0}^{j}\right) \subset C(\Omega)$ such that $0 \leq u_{0}^{j} \leq u_{0}$ and $u_{0}^{j} \rightarrow u_{0}$ a.e.. We keep the notation $u^{\varepsilon}$ for the solution of $\left(P_{\varepsilon}\right)$ with initial condition $u_{0}$, and let $u^{\varepsilon, j}$ denote the solution of $\left(P_{\varepsilon}\right)$ with initial condition $u_{0}^{j}$. By comparison (which is possible because the nonlinearity in $\left(P_{\varepsilon}\right)$ is smooth) $u^{\varepsilon, j}(t) \leq u^{\varepsilon}(t)$ for all $t \geq 0$. First let $\varepsilon \rightarrow 0$ and then $j \rightarrow \infty$ to conclude that $u_{0} \leq \hat{u}_{0}$ a.e. in $\Omega$, so that with (72) we get the equality $\hat{u}_{0}=u_{0}$ a.e. in $\Omega$.

\section{Solutions With Positive initial Data}

Proof of Lemma 1.9. We will find a positive subsolution $\underline{u}$, more precisely, a function $\underline{u}$ satisfying

$$
\left\{\begin{aligned}
\underline{u}_{t}-\Delta \underline{u}+\frac{1}{\underline{u}^{\beta}} & \leq 0 & & \text { in } \Omega \times(0, T), \\
\underline{u}(0) & \leq u_{0} & & \text { in } \Omega, \\
\underline{u} & =0 & & \text { on } \partial \Omega \times(0, T),
\end{aligned}\right.
$$


whenever the initial condition satisfies $u_{0} \geq c \delta^{\nu}$ for some $c>0$ and $1<\nu<\frac{2}{1+\beta}$. Then $\underline{u}$ is also a subsolution of $\left(P_{\varepsilon}\right.$ for any $\varepsilon>0$, and by comparison the solution $u^{\varepsilon}$ of $P_{\varepsilon}$ satisfies $u^{\varepsilon}(t) \geq \underline{u}(t)$ for all $t \in(0, T)$. Thus, when we let $\varepsilon \rightarrow 0$ we discover $u(t) \geq \underline{u}(t)$ for all $t \in(0, T)$.

We take $\underline{u}$ of the form $\underline{u}=\varepsilon \varphi_{1}^{\nu} e^{-A t}$ and $T=\frac{\log 2}{A}$, where $\varphi_{1}$ is the first positive eigenfunction of the Laplacian with Dirichlet boundary condition. We take $\varepsilon>0$ small so that $\underline{u}(0)=\varepsilon \varphi_{1}^{\nu} \leq u_{0}$. The constant $A$ will be chosen later. Since $e^{\beta T}=2^{\frac{\beta}{A}}$, we get

$$
\begin{aligned}
\underline{u}_{t}-\Delta \underline{u}+\frac{1}{\underline{u}^{\beta}} & =-A \varepsilon e^{-A t} \varphi_{1}^{\nu}+\varepsilon e^{-A t}\left(\lambda_{1} \nu \varphi_{1}^{\nu}-\nu(\nu-1) \varphi_{1}^{\nu-2}\left|\nabla \varphi_{1}\right|^{2}\right)+\varepsilon^{-\beta} e^{A \beta t} \varphi_{1}^{-\nu \beta} \\
& \leq-\frac{1}{2} A \varepsilon \varphi_{1}^{\nu}+\varepsilon \lambda_{1} \nu \varphi_{1}^{\nu}+\varphi_{1}^{-\nu \beta}\left(\varepsilon^{-\beta} 2^{\beta}-\frac{1}{2} \varepsilon \nu(\nu-1) \varphi_{1}^{\nu-2+\nu \beta}\left|\nabla \varphi_{1}\right|^{2}\right) \\
& \leq\left(-\frac{1}{2} A+\lambda_{1} \nu\right) \varepsilon \varphi_{1}^{\nu}+\varphi_{1}^{-\nu \beta}\left(2 \varepsilon^{-\beta}-\frac{1}{2} \varepsilon \nu(\nu-1) \varphi_{1}^{\nu-2+\nu \beta}\left|\nabla \varphi_{1}\right|^{2}\right) .
\end{aligned}
$$

Let $\Omega_{\sigma}=\{x \in \Omega$ : $\operatorname{dist}(x, \partial \Omega)<\sigma\}$. We can choose $\sigma>0$ small enough so that

$$
2 \varepsilon^{-\beta}-\frac{1}{2} \varepsilon \nu(\nu-1) \varphi_{1}^{\nu-2+\nu \beta}\left|\nabla \varphi_{1}\right|^{2}<0 \quad \text { on } \Omega_{\sigma}
$$

because the exponent of $\varphi_{1}$ is $\nu-2+\nu \beta<0$ ( since $\left.\nu<\frac{2}{1+\beta}\right)$.

Then choose $A$ large enough so that

$$
\left(-\frac{1}{2} A+\lambda_{1} \nu\right) \varepsilon \varphi_{1}^{\nu}+2 \varphi_{1}^{-\nu \beta} \varepsilon^{-\beta} \leq 0 \quad \text { on } \Omega-\Omega_{\sigma} .
$$

Proof of Proposition 1.12. We just need to verify the property for smooth functions, the conclusion follows by density. First let us recall Hardy's inequality: there exists $C=C(\Omega)$ such that

$$
\int_{\Omega} \frac{\varphi^{2}}{\delta^{2}} d x \leq C \int_{\Omega}|\nabla \varphi|^{2} d x \quad \forall \varphi \in C_{0}^{\infty}(\Omega) .
$$

For $\psi, \varphi \in C_{0}^{\infty}(\Omega)$ we have

$$
\begin{aligned}
\int_{\Omega} S(t)\left(\delta^{-m} \psi\right) \varphi & =\int_{\Omega} \psi \delta^{-m} S(t) \varphi \\
& \leq\left(\int_{\Omega} \delta^{-2 m}(S(t) \varphi)^{2}\right)^{1 / 2}\|\psi\|_{L^{2}(\Omega)} \\
& \leq\left(\int_{\Omega} \delta^{-2}(S(t) \varphi)^{2}\right)^{m / 2}\|S(t) \varphi\|_{L^{2}(\Omega)}^{1-m}\|\psi\|_{L^{2}(\Omega)}
\end{aligned}
$$

Observe now that $S(t) \varphi \in H_{0}^{1}(\Omega)$ and therefore we can apply (76):

$$
\int_{\Omega} S(t)\left(\delta^{-m} \psi\right) \varphi \leq C\|S(t) \varphi\|_{H_{0}^{1}(\Omega)}^{m}\|S(t) \varphi\|_{L^{2}(\Omega)}^{1-m}\|\psi\|_{L^{2}(\Omega)}
$$

and by the smoothing effect of the heat semigroup $S(t)$ from $L^{2}(\Omega)$ to $H_{0}^{1}(\Omega)$ we find that

$$
\begin{aligned}
\int_{\Omega} S(t)\left(\delta^{-m} \psi\right) \varphi & \leq C\left(t^{-1 / 2}\|S(t) \varphi\|_{L^{2}(\Omega)}\right)^{m}\|S(t) \varphi\|_{L^{2}(\Omega)}^{1-m}\|\psi\|_{L^{2}(\Omega)} \\
& =C t^{-m / 2}\|S(t) \varphi\|_{L^{2}(\Omega)}\|\psi\|_{L^{2}(\Omega)}
\end{aligned}
$$

which yields the desired result. 
The following singular version of the Gronwall inequality (see [BC]), will be used in the proof of Lemma 1.11 and Theorem 1.6

Lemma 5.1. Let $T>0, A \geq 0,0 \leq a, b \leq 1$ and let $f$ be a nonnegative function with $f \in L^{p}(0, T)$ for some $p>1$ such that $p^{\prime} \max (a, b)<1$, where $1=\frac{1}{p}+\frac{1}{p^{\prime}}$. Consider a nonnegative function $\varphi \in L^{\infty}(0, T)$ such that

$$
\varphi(t) \leq A t^{-a}+\int_{0}^{t}(t-s)^{-b} f(s) \varphi(s) d s, \quad \text { for almost all } t \in[0, T] .
$$

Then there exists $C$, depending only on $T, a, b, p$ and $\|f\|_{L^{p}}$ such that

$$
\varphi(t) \leq A C t^{-a}
$$

for almost all $t \in[0, T]$.

Proof of Lemma 1.11. Step 1. First let us prove that $u(t) \leq v(t)$ for $t \in[0, T]$ under the additional assumption that $u>0$ a.e. in $\Omega \times(0, T)$. Then

$$
u(t) \leq S(t) u_{0}-\int_{0}^{t} S(t-s)(g(u(s))) d s \quad t \in[0, T],
$$

and

$$
v(t) \geq S(t) v_{0}-\int_{0}^{t} S(t-s)(g(v(s))) d s \quad t \in[0, T] .
$$

Let $A>0$. Observe that the previous relations imply that

$$
e^{A t} u(t) \leq S(t) u_{0}+\int_{0}^{t} S(t-s)\left(e^{A s}(A u(s)-g(u(s)))\right) d s
$$

and

$$
e^{A t} v(t) \leq S(t) v_{0}+\int_{0}^{t} S(t-s)\left(e^{A s}(A v(s)-g(v(s)))\right) d s .
$$

Let $w=u-v$, so that $w$ satisfies

$$
e^{A t} w(t) \leq \int_{0}^{t} S(t-s)\left(e^{A s}(A w(s)+g(v(s))-g(u(s))) d s .\right.
$$

By convexity of the function $u \mapsto u^{-\beta}$ we obtain the estimate

$$
g(v)-g(u)=v^{-\beta}-u^{-\beta}-f(v)+f(u) \leq \beta v^{-1-\beta}(u-v)+k|u-v|
$$

for a large constant $k>0$.

Using the lower bound $v(s) \geq c \delta^{\nu}$ we thus obtain

$$
g(v(s))-g(u(s)) \leq C \delta^{-\nu(1+\beta)} w^{+}(s)+k|w(s)|
$$

so that

$$
g(v(s))-g(u(s))+A w(s) \leq C \delta^{-\nu(1+\beta)} w^{+}(s)
$$

if one chooses $A$ large enough. Combining with (77) we see that

$$
e^{A t} w(t) \leq C \int_{0}^{t} S(t-s)\left(e^{A s} \delta^{-\nu(1+\beta)} w^{+}(s)\right) d s .
$$

Since the right-hand side is a nonnegative function, we also obtain

$$
e^{A t} w^{+}(t) \leq c \int_{0}^{t} S(t-s)\left(e^{A s} \delta^{-\nu(1+\beta)} w^{+}(s)\right) d s .
$$


Let $\gamma=\nu(1+\beta)-1$ so that $0<\gamma<1$. Multiplying the previous relation by $\delta^{-\gamma}$ and computing the $L^{2}$ norm of the resulting functions we get

$$
e^{A t}\left\|\delta^{-\gamma} w^{+}(t)\right\|_{L^{2}} \leq C \int_{0}^{t}\left\|\delta^{-\gamma} S(t-s)\left(\delta^{-\nu(1+\beta)} w^{+}(s)\right)\right\|_{L^{2}} e^{A s} d s .
$$

Let $\psi=S(t-s)\left(\delta^{-\nu(1+\beta)} w^{+}(s)\right)$. By Hölder's inequality

$$
\left\|\delta^{-\gamma} \psi\right\|_{L^{2}}^{2}=\int \frac{\psi^{2}}{\delta^{-2 \gamma}} \leq\left(\int \frac{\psi^{2}}{\delta^{-2}}\right)^{\gamma}\left(\int \psi^{2}\right)^{1-\gamma}
$$

and by Hardy's inequality (76)

$$
\left\|\delta^{-\gamma} \psi\right\|_{L^{2}} \leq C\|\nabla \psi\|_{L^{2}}^{\gamma}\|\psi\|_{L^{2}}^{1-\gamma} .
$$

By the smoothing effect of the heat semigroup

$$
\begin{aligned}
\|\nabla \psi\|_{L^{2}} & =\left\|\nabla S(t-s)\left(\delta^{-\nu(1+\beta)} w^{+}(s)\right)\right\|_{L^{2}} \\
& \leq C(t-s)^{-1 / 2}\left\|S\left(\frac{t-s}{2}\right)\left(\delta^{-\nu(1+\beta)} w^{+}(s)\right)\right\|_{L^{2}}
\end{aligned}
$$

so that

$$
\left\|\delta^{-\gamma} S(t-s)\left(\delta^{-\nu(1+\beta)} w^{+}(s)\right)\right\|_{L^{2}} \leq C(t-s)^{-\gamma / 2}\left\|S\left(\frac{t-s}{2}\right)\left(\delta^{-\nu(1+\beta)} w^{+}(s)\right)\right\|_{L^{2}} .
$$

Substituting in (78) we find that

$$
e^{A t}\left\|\delta^{-\gamma} w^{+}(t)\right\|_{L^{2}} \leq C \int_{0}^{t}(t-s)^{-\gamma / 2} e^{A s}\left\|S\left(\frac{t-s}{2}\right)\left(\delta^{-1} \delta^{-\gamma} w^{+}(s)\right)\right\|_{L^{2}} d s,
$$

and by Lemma 1.12

$$
e^{A t}\left\|\delta^{-\gamma} w^{+}(t)\right\|_{L^{2}} \leq C \int_{0}^{t}(t-s)^{-\gamma / 2-1 / 2} e^{A s}\left\|\delta^{-\gamma} w^{+}(s)\right\|_{L^{2}} d s .
$$

Observe that $\gamma / 2+1 / 2<1$. By Lemma 5.1

$$
e^{A t}\left\|\delta^{-\gamma} w^{+}(t)\right\|_{L^{2}}=0 \quad t \in[0, T]
$$

which is the desired conclusion.

Step 2. We proceed now with the general case. Let $z$ be the solution to $(P)$ with $z(0)=v(0) \geq c \delta^{\nu}$. By Lemma 1.9 there is a constant $c^{\prime}>0$ and $0<t_{1} \leq T$ such that $z(t) \geq c^{\prime} \delta^{\nu}$ for $t \in\left[0, t_{1}\right]$. We claim that $z(t) \geq u(t)$ for $t \in[0, T]$. In fact, let $z_{\varepsilon}$ be the solution of $\left[P_{\varepsilon}\right]$ with $z^{\varepsilon}(0)=z(0)=v(0)$. Since $u$ is the subsolution of $(\underline{P})$, it is also a subsolution to $\left(P_{\varepsilon}\right)$. We obtain $u(t) \leq z^{\varepsilon}(t)$ for every $t \in[0, T]$, since $u(0) \leq z^{\varepsilon}(0)$. Letting $\varepsilon \rightarrow 0$ we obtain $u(t) \leq z(t)$ for $t \in[0, T]$. On the other hand, Step 1 applied with $z$ instead of $u$ implies that $z(t) \leq v(t)$ for $t \in\left[0, t_{1}\right]$, since $v(0)=z(0)$. Therefore, $u(t) \leq v(t)$ for $t \in\left[0, t_{1}\right]$. Repeating the previous reasoning in $[t, 2 t]$ we obtain $u(t) \leq v(t)$ for every $t \in\left[0,2 t_{1}\right]$. By induction we obtain the conclusion.

\section{Asymptotic Behavior: POSITIVE Versus VANishing SOLUtions}

Proof of Theorem [1.6. We denote by $\left(E \hat{\lambda}_{+\varepsilon}\right)$ the problem $(E)$ with parameter $\lambda+\varepsilon$ (instead of $\lambda$ ). We are going to prove that for every $\varepsilon>0$ problem $\left(E_{+}\right)$has a solution which is positive a.e. 
Suppose that $(P)$ has a solution $u$ which is positive a.e. in $\Omega \times(0, \infty)$.

Step 1. We define by $\left(P \lambda_{+\varepsilon}\right)$ to be the parabolic problem $(\underline{P})$ with $g(u)$ replaced by $-u^{\beta}+(\lambda+\varepsilon) f(u)$. The problem $\left(\underline{P}_{+\varepsilon}\right)$ has a global positive solution $\widetilde{u}(t)$ which is nonincreasing in $t$.

Let $\zeta$ be the solution of

$$
\left\{\begin{aligned}
\zeta_{t}-\Delta \zeta & =f(u+\varepsilon \zeta) & & \text { in } \Omega \times(0, \infty), \\
\zeta & =0 & & \text { on } \partial \Omega \times(0, \infty), \\
\zeta(0) & =0 & & \text { in } \Omega .
\end{aligned}\right.
$$

Then $u+\varepsilon \zeta$ satisfies

$(u+\varepsilon \zeta)_{t}-\Delta(u+\varepsilon \zeta)=-\frac{1}{u^{\beta}}+\lambda f(u)+\varepsilon f(u+\varepsilon \zeta) \leq-\frac{1}{(u+\varepsilon \zeta)^{\beta}}+(\lambda+\varepsilon) f(u+\varepsilon \zeta)$

and therefore it is a positive subsolution of

$$
\left\{\begin{aligned}
u_{t}-\Delta u & =\bar{g}(u) & & \text { in } \Omega \times(0, T), \\
u(x, t) & =0 & & \text { on } \partial \Omega \times(0, T), \\
u(x, 0) & =u_{0}(x) & & \text { in } \Omega,
\end{aligned}\right.
$$

where

$$
\bar{g}(u)=-u^{-\beta}+(\lambda+\varepsilon) f(u) .
$$

Remark that $\zeta(t) \geq c(t) \delta$ (see [BCMR]), where $c:(0, \infty) \rightarrow(0, \infty)$ can be taken to be continuous. By shifting time, we can assume that $\inf _{t \in(0, T)} c(t)>0$ for any $T>0$.

Take $\widetilde{u}_{0}$ a supersolution of $\left(E \vec{\lambda}_{+\varepsilon}\right)$ (which is also a supersolution of (799) such that $\widetilde{u}_{0} \geq u(0)$. Then the initial boundary value problem (79) has a global solution $\widetilde{u}$ such that $u(t)+\varepsilon \zeta \leq \widetilde{u}(t) \leq \widetilde{u}_{0}$.

We prove now that $\widetilde{u}(t)$ is nonincreasing in $t$. Indeed, fix $T>0$, let $h>0$ and set $v(t)=\widetilde{u}(t+h)-\widetilde{u}(t)$. Then $v$ satisfies

$$
\begin{aligned}
v(t) & =S(t) v(0)+\int_{0}^{t} S(t-s)(\bar{g}(\widetilde{u}(s+h))-\bar{g}(\widetilde{u}(s))) d s \\
& \leq \int_{0}^{t} S(t-s)(c(s) v(s)) d s
\end{aligned}
$$

where

$$
c(s)=\frac{\bar{g}(\widetilde{u}(s+h))-\bar{g}(\widetilde{u}(s))}{\widetilde{u}(s+h)-\widetilde{u}(s)} \leq \bar{g}^{\prime}(\widetilde{u}(s)) \leq C \delta^{-1-\beta}
$$

for some constant $C>0$, because $\widetilde{u}(s) \geq c \delta$ for all $s \in[0, T]$. Then, as in the proof of Lemma 1.11, we get

$$
\begin{aligned}
\left\|\delta^{-\beta} v^{+}(t)\right\|_{L^{2}} & \leq C \int_{0}^{t}\left\|\delta^{-\beta} S(t-s)\left(\delta^{-1-\beta} v^{+}(s)\right)\right\|_{L^{2}} d s \\
& \leq C \int_{0}^{t}(t-s)^{-\beta / 2}\left\|S\left(\frac{t-s}{2}\right)\left(\delta^{-1-\beta} v^{+}(s)\right)\right\|_{L^{2}} d s \\
& \leq C \int_{0}^{t}(t-s)^{-(\beta+1) / 2}\left\|\delta^{-\beta} v^{+}(s)\right\|_{L^{2}} d s .
\end{aligned}
$$

Since $\frac{\beta+1}{2}<1$ by the singular Gronwall Lemma 5.1 we conclude that $v^{+}(t) \leq 0$ for all $t \in[0, T]$. But $T>0$ was arbitrary so that $v^{+}(t) \leq 0$ for all $t \geq 0$. 
Step 2. By the previous step the limit $\widetilde{u}(t) \rightarrow w$ as $t \rightarrow \infty$ exists, we claim that $w$ is a solution of $\left(E_{\lambda+\varepsilon}\right)$.

Integrating (79) on $\Omega \times(t, t+1)$ we find

$$
\int_{\Omega}(\widetilde{u}(t+1)-\widetilde{u}(t)) d x+\int_{t}^{t+1} \int_{\Omega} \widetilde{u}(s)^{-\beta} d x d s \leq(\lambda+\varepsilon) \int_{t}^{t+1} \int_{\Omega} f(\widetilde{u}(s)) d x d s,
$$

thus

$$
\int_{\Omega} \widetilde{u}(t)^{-\beta} d x \leq(\lambda+\varepsilon) \int_{t}^{t+1} \int_{\Omega} f(\widetilde{u}(s)) d x d s+\int_{\Omega} \widetilde{u}(t) d x \leq C,
$$

where $C$ is independent of $t$. This shows that $\widetilde{u}(t)^{-\beta}$ is bounded in $L^{1}(\Omega)$, and since it is monotone increasing, it converges to $w^{-\beta}$ as $t \rightarrow \infty$ and $w^{-\beta} \in L^{1}(\Omega)$. find

Take $\varphi \in C^{2}(\bar{\Omega})$ with $\varphi=0$ on $\partial \Omega$. Then integrating (79) on $\Omega \times(t, t+1)$ we

$$
\int_{\Omega}(\widetilde{u}(t+1)-\widetilde{u}(t)) \varphi+\int_{t}^{t+1} \int_{\Omega} \widetilde{u}(s)(-\Delta \varphi) d x d s=\int_{t}^{t+1} \int_{\Omega} \bar{g}(\widetilde{u}(s)) \varphi d x d s .
$$

Letting $t \rightarrow \infty$ we deduce that $w$ is a solution of $\left(E_{\lambda}+\varepsilon\right)$.

Proof of Theorem 1.7. Observe that $w$ is a subsolution of $\left(P_{\varepsilon}\right)$ and therefore $u^{\varepsilon}(t) \geq$ $w$ for all $t \geq 0$. We will use this to show that $u=\lim _{\varepsilon \rightarrow 0} u^{\varepsilon}$ solves (P) in the sense of (3) - (4). The reason is that we have an upper bound on $g_{\varepsilon}\left(u^{\varepsilon}\right)$ and we can use dominated convergence to pass to the limit. In fact, let $\varphi \in C^{2}(\bar{\Omega} \times[0, T])$ with $\left.\varphi\right|_{\partial \Omega \times(0, T)}=0,\left.\varphi\right|_{\Omega \times\{T\}}=0$. Then

$$
\int_{\Omega} u_{0} \varphi(0) d x+\int_{0}^{T} \int_{\Omega}\left(\varphi_{t}+\Delta \varphi\right) u^{\varepsilon}-g_{\varepsilon}\left(u^{\varepsilon}\right) \varphi d x d t=0 .
$$

Since $u^{\varepsilon}(t) \geq w>0$ a.e., we have $\left|g_{\varepsilon}\left(u^{\varepsilon}\right)\right| \leq w^{-\beta}+f(w) \in L^{1}(\Omega)$. So in fact we can apply dominated convergence and deduce that

$$
\int_{\Omega} u_{0} \varphi(0) d x+\int_{0}^{T} \int_{\Omega}\left(\varphi_{t}+\Delta \varphi\right) u-g(u) \varphi d x d t=0 .
$$

The lower bound $u(t) \geq c(t) \delta$ for all $t>0$, where $c:(0, \infty) \rightarrow(0, \infty)$ is continuous follows from the fact that $v(t)=w+z(t)$ is a subsolution of (P) where $z=$ $S(t)\left(u_{0}-w\right)$, i.e. the solution of

$$
\left\{\begin{aligned}
z_{t} & =\Delta z & & \text { in } \Omega \times(0, \infty), \\
z & =0 & & \text { on } \partial \Omega \times(0, \infty), \\
z(0) & =u_{0}-w & & \text { in } \Omega .
\end{aligned}\right.
$$

In fact,

$$
v_{t}-\Delta v+g(v)=\Delta w+g(w+z)=g(w+z)-g(w) \leq 0,
$$

because $g$ is nonincreasing.

Finally, recall that since $u_{0} \not \equiv w$, we have $z(t) \geq c(t) \delta$ (this is standard, see BCMR]).

Proof of Corollary 1.8 Let $\beta \geq 1$, and for the sake of contradiction assume that there is a global positive solution $u$ of $(P)$.

Let $Y$ be the solution to

$$
\left\{\begin{aligned}
-\Delta Y=1 & \text { in } \Omega \\
Y=0 & \text { on } \partial \Omega .
\end{aligned}\right.
$$


Then observe that $u(t) \leq K Y$ for some constant $K$ independent of $t$, because for large $K, \bar{u}=K Y$ is a supersolution of $(P)$, and one can argue as in Lemma 1.11.

Set $v(t)=u(t) / K$, so that $0<v(t) \leq 1$ and satisfies

$$
\left\{\begin{aligned}
v_{t}-\Delta v+\frac{1}{K^{1+\beta}} \frac{1}{v^{\beta}} & =\lambda \tilde{f}(v) & & \text { in } \Omega \times(0, \infty), \\
v & =0 & & \text { on } \partial \Omega \times(0, \infty),
\end{aligned}\right.
$$

where $\tilde{f}(v)=\frac{1}{K} f(K v)$. Therefore, for any $0<\beta_{1}<1 v$ is a positive global subsolution of

$$
\left\{\begin{aligned}
u_{t}-\Delta u+\frac{1}{K^{1+\beta}} \frac{1}{u^{\beta_{1}}} & =\lambda \tilde{f}(u) & & \text { in } \Omega \times(0, \infty), \\
u & =0 & & \text { on } \partial \Omega \times(0, \infty), \\
u(0) & =C \delta & & \text { in } \Omega,
\end{aligned}\right.
$$

where $C>0$ is fixed large enough. Hence (80) has a global positive solution, and by Theorem 1.6 the elliptic problem

$$
\left\{\begin{aligned}
-\Delta w+\frac{1}{K^{1+\beta}} \frac{1}{w^{\beta_{1}}} & =\lambda \tilde{f}(w) & & \text { in } \Omega, \\
w & =0 & & \text { on } \partial \Omega
\end{aligned}\right.
$$

has a solution $w$ which is positive a.e. and such that $w \leq C \delta$ with $C$ independent of $\beta_{1}$. But this is impossible, because

$$
\int_{\Omega} w^{-\beta_{1}} \leq \lambda \int_{\Omega} \tilde{f}(w) \leq C,
$$

and $C$ is independent of $\beta_{1}$. This is obtained, formally, by integrating (81) over $\Omega$, but a rigorous argument can be found in DM1. Letting $\beta_{1} \nearrow 1$ in (82) we reach a contradiction.

\section{ACKNOWLEDGMENTS}

J.D. was supported by FONDECYT 1020815. The authors also kindly acknowledge the support by FAPESP 03/02333-5, CMM-Universidad de Chile and Pronex.

\section{REFERENCES}

[AK] A. Acker and B. Kawohl, Remarks on quenching. Nonlinear Anal. 13 (1989), 53-61. MR0973368 (90c:35007)

[A] R. Aris, The Mathematical Theory of Diffusion and Reaction in Permeable Catalysts. Clarendon Press, Oxford, 1975.

[B] H. T. Banks, Modeling and control in the biomedical sciences. Lecture Notes in Biomathematics, Vol. 6. Springer-Verlag, Berlin-New York, 1975. MR0401201 (53:5030)

[BC] H. Brezis and T. Cazenave, A nonlinear heat equation with singular initial data. J. Anal. Math. 68 (1996), 277-304. MR1403259|(97f:35092)

[BCMR] H. Brezis, T. Cazenave, Y. Martel, and A. Ramiandrisoa, Blow-up for $u_{t}-\Delta u=g(u)$ revisited. Adv. Differential Equations 1 (1996), 73-90. MR1357955 (96i:35063)

[CK] C.Y. Chan and M.K. Kwong, Quenching phenomena for singular nonlinear parabolic equations. Nonlinear Anal. 12 (1988), 1377-1383. MR0972406 (90a:35110)

[Da] J. Dávila, Global regularity for a singular equation and local $H^{1}$ minimizers of a nondifferentiable functional. Commun. in Contemporary Mathematics 6 (2004), 165-193. MR.2048779

[DM1] J. Dávila and M. Montenegro, Positive versus free boundary solutions to a singular equation. J. Anal. Math. 90 (2003), 303-335. MF2001074 
[DM2] J. Dávila and M. Montenegro, A singular equation with positive and free boundary solutions. RACSAM Rev. R. Acad. Cienc. Exactas Fís. Nat. Ser. A Mat. 97 (2003), 107-112. MR2037228 (2004m:35097)

[DL] K. Deng and H.A. Levine On the blow up of $u_{t}$ at quenching. Proc. Amer. Math. Soc. 106 (1989), 1049-1056. MR0969520 (90f:35030)

[D] J. I. Díaz, Nonlinear partial differential equations and free boundaries. Vol. I. Pitman (Advanced Publishing Program), Boston, MA, 1985, Elliptic equations. MR0853732 (88d:35058)

[FH] M. Fila and J. Hulshof, A note on the quenching rate. Proc. Amer. Math. Soc. 112 (1991), no. 2, 473-477. MR1055772 (92a:35090)

[FHQ] M. Fila, J. Hulshof and P. Quittner, The quenching problem on the $N$-dimensional ball. Nonlinear diffusion equations and their equilibrium states, 3 (Gregynog, 1989), 183-196, Progr. Nonlinear Differential Equations Appl., 7, Birkhäuser Boston, Boston, MA, 1992. MR:1167839 (93c:35078)

[FK] M. Fila and B. Kawohl, Is quenching in infinite time possible?. Quart. Appl. Math. 48 (1990), 531-534. MR 1074968 (91h:35054)

[FK2] M. Fila and B. Kawohl, Asymptotic analysis of quenching problems. Rocky Mountain J. Math. 22 (1992), 563-577. MR1180720|(93h:35093)

[FKL] M. Fila, B. Kawohl and H.A. Levine, Quenching for quasilinear equations. Comm. Partial Differential Equations 17 (1992), 593-614. MR1163438 (93f:35101)

[FLV] M. Fila, H.A. Levine and J.L. Vázquez, Stabilization of solutions of weakly singular quenching problems. Proc. Amer. Math. Soc. 119 (1993), 555-559. MR1174490 (93k:35146)

[FG] S. Filippas and J. Guo, Quenching profiles for one-dimensional semilinear heat equations. Quart. Appl. Math. 51 (1993), 713-729. MR1247436 (95b:35029)

[GL] C. Gui and F.H. Lin, Regularity of an elliptic problem with a singular nonlinearity. Proc. Roy. Soc. Edinburgh Sect. A 123 (1993), 1021-1029. MR1263903 (94m:35115)

$[\mathrm{Gu}]$ J. Guo, On the quenching rate estimate. Quart. Appl. Math. 49 (1991), 747-752. MR1134750 (92j:35097)

[K] H. Kawarada, On solutions of initial-boundary problem for $u_{t}=u_{x x}+1 /(1-u)$. Publ. Res. Inst. Math. Sci. 10 (1974/75), 729-736. MR0385328 (52:6192)

[LSU] O. A. Ladyzenskaja, V. A. Solonnikov and N. N. Uralceva, Linear and quasilinear equations of parabolic type. Translations of Mathematical Monographs, Vol. 23, American Mathematical Society, Providence, R.I. 1967. MR 0241822 (39:3159b)

[L] H.A. Levine, Quenching and beyond: a survey of recent results. Nonlinear mathematical problems in industry, II (Iwaki, 1992), 501-512, GAKUTO Internat. Ser. Math. Sci. Appl., 2, Gakkōtosho, Tokyo, 1993. MR1370487 (96h:35089)

[P] D. Phillips, Existence of solutions of quenching problems. Appl. Anal. 24 (1987), 253264. MR0907341 (88k:35101)

Departamento de Ingeniería Matemática, CMM (UMR CNRS), Universidad de Chile, Casilla $170 / 3$, Correo 3 , Santiago, Chile

E-mail address: jdavila@dim.uchile.cl

Departamento de Matemática, Universidade Estadual de Campinas, imeCC, Caixa Postal 6065, CEP 13084-970, Campinas, SP, Brasil

E-mail address: msm@ime.unicamp.br 